\title{
Next-to-next-to-leading order spin-orbit effects in the gravitational wave flux and orbital phasing of compact binaries
}

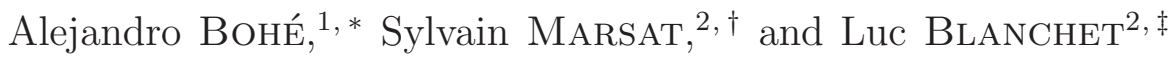 \\ ${ }^{1}$ Departament de Fúsica, Universitat de les Illes Balears, \\ Crta. Valldemossa km 7.5, E-07122 Palma, Spain \\ ${ }^{2} \mathcal{G} \mathbb{R} \varepsilon \mathbb{C O}$ Institut d'Astrophysique de Paris - UMR 7095 du CNRS, \\ Université Pierre \& Marie Curie, $98^{\text {bis }}$ boulevard Arago, 75014 Paris, France
}

(Dated: September 6, 2018)

\begin{abstract}
We compute the next-to-next-to-leading order spin-orbit contributions in the total energy flux emitted in gravitational waves by compact binary systems. Such contributions correspond to the post-Newtonian order 3.5PN for maximally spinning compact objects. Continuing our recent work on the next-to-next-to-leading spin-orbit terms at 3.5PN order in the equations of motion, we obtain the spin-orbit terms in the multipole moments of the compact binary system up to the same order within the multipolar post-Newtonian wave generation formalism. Our calculation of the multipole moments is valid for general orbits and in an arbitrary frame; the moments are then reduced to the center-of-mass frame and the resulting energy flux is specialized to quasi-circular orbits. The test-mass limit of our final result for the flux agrees with the already known Kerr black hole perturbation limit. Furthermore the various multipole moments of the compact binary reduce in the one-body case to those of a single boosted Kerr black hole. We briefly discuss the implications of our result for the gravitational-wave flux in terms of the binary's phase evolution, and address its importance for the future detection and parameter estimation of signals in gravitational wave detectors.
\end{abstract}

PACS numbers: 95.35.+d,95.36.+x,04.50.Kd

\footnotetext{
*alejandro.bohe@uib.es

$\dagger$ marsat@iap.fr

†lanchet@iap.fr
} 


\section{INTRODUCTION}

Previous works [1, 2] 1 have derived the spin-orbit effects in the equations of motion of compact binary systems (made of two black holes or neutron stars) at the next-to-next-toleading order beyond the dominant level. Such an order corresponds to the post-Newtonian $(\mathrm{PN})$ order $3.5 \mathrm{PN} \sim 1 / c^{7}$ in the case of maximally spinning compact objects, i.e. $2 \mathrm{PN}$ beyond the leading spin-orbit effect at order $1.5 \mathrm{PN} \sim 1 / c^{3}$. The present paper will continue Papers I \& II by investigating the gravitational radiation field of compact binaries, notably the total gravitational wave energy flux and orbital phase evolution, up to the same nextto-next-to-leading 3.5PN level.

Including spin effects, and most importantly spin-orbit effects which are linear in spins, in the templates of gravitational waves emitted by compact binaries is of crucial importance for the accurate data analysis of the advanced ground-based as well as future space-based gravitational wave detectors. Astrophysical stellar-size black holes [3 7] as well as supermassive black holes [8-10] have spins, and the spins will affect the gravitational waves of black hole binaries through a modulation of their amplitude, phase and frequency (including the precession of the orbital plane in the case of non-aligned spins, see e.g. Refs. [11 13]).

The leading spin-orbit and spin-spin contributions in the equations of motion have been obtained using various methods [14 -19]; the next-to-leading corrections are also known both for the spin-orbit [20 24] and spin-spin terms [25 29]; next-to-next-to-leading spin-orbit corrections have been derived in Refs. [30, 31] and in Papers I \& II. Concerning the radiation field of compact binaries, the leading spin-orbit and spin-spin terms are known [16, 17]; the next-to-leading spin-orbit terms at order 2.5PN $\sim 1 / c^{5}$ were first obtained in Ref. [21] after a previous attempt in [32]; the $3 \mathrm{PN} \sim 1 / c^{6}$ spin-orbit contribution including the tail integrals were computed in [33], after intermediate results at the same order (but including spinspin terms) were given in [34]; finally, the next-to-next-to-leading order contributions in the multipole moments and the energy flux, corresponding to $3.5 \mathrm{PN} \sim 1 / c^{7}$, is the topic of the present paper and has never been addressed before.

Following the previous investigations [21, 33] we shall apply the so-called multipolar post-Newtonian approach to gravitational radiation. This approach has been extensively developped over the years (see [35] for a review). It combines a mixed post-Minkowskian and multipolar expansion for the gravitational field in the exterior of a general source having compact support [36 39], with a matching to the post-Newtonian expansion of the inner field in the near zone of a post-Newtonian source [40 44]. The gravitational waveform and various fluxes like the energy flux (or gravitational "luminosity") are expanded in a series of radiative multipole moments, that are then related to appropriate source-rooted multipole moments, expressed as integrals over the matter and gravitational fields in the source. The time derivatives of the multipole moments are performed using the equations of motion of the source, which must thus be known beforehand with the same accuracy as the one aimed for in the radiation field.

Though the formalism can be applied to any post-Newtonian source, it does require a model for the source. In the case of compact binaries, the compact objects are described by point-like particles characterized only by their masses and their spins. The appropriate model in this context is an effective "pole-dipole" description, based on a stress-energy tensor made of a monopole or mass term involving delta functions, and a dipole or spin term made of gradients of delta functions. The pole-dipole description of spinning particles has been

\footnotetext{
${ }^{1}$ Hereafter these works will be referred to as Papers I \& II respectively.
} 
developed by many authors [45 55]. The model is to be suplemented by an ultra-violet (UV) regularization in order to remove the infinite self-field of the point masses, see e.g. [56, 57]. In Papers I \& II and the present paper, we use (and present justifications for that use at the aimed level of accuracy) the Hadamard "partie finie" regularization [58], together with the Gel'Fand-Shilov prescription for distributional derivatives [59], equivalent to Schwartz distributional derivatives [60]. Explicit expressions for the waveform and flux can then be obtained in terms of the source's positions and velocities, and these can finally be converted into gauge-invariant quantities that are directly in use for building the gravitational wave templates.

The plan of this paper is as follows. The Section II is devoted to the general wave generation formalism and the Section III to the application to spinning compact binaries. We give the required formulas in Sec. II A for the various types of multipole moments (radiative, canonical and source), and in Sec. IIB for the general post-Newtonian solution we employ. The complete results for the source multipole moments at next-to-next-to-leading spin-orbit level are presented in Sec. IIIA, while we obtain in Sec. IIIB the next-to-next-to-leading energy flux and orbital phase evolution. We also present a numerical estimate of the new terms in Sec. IIIB. In Appendix $\mathrm{A}$ we give alternative expressions of the source terms to be inserted into the source multipole moments. In Appendix B we check the agreement with the so-called boosted Kerr black hole limit, obtained when the mass and spin of one of the two black holes are set to be exactly zero.

\section{GRAVITATIONAL WAVE GENERATION FORMALISM}

\section{A. Radiative and source multipole moments}

The gravitational waveform, generated by an isolated source described by a stress-energy tensor with compact support, is the transverse-tracefree (TT) projection of the metric deviation, say $h_{i j}^{\mathrm{TT}} \equiv\left(g_{i j}-\delta_{i j}\right)^{\mathrm{TT}}$. It is defined in a suitable radiative coordinate system $X^{\mu}=(c T, \mathbf{X})$, at the leading-order $1 / R$ when the distance $R=|\mathbf{X}|$ to the source tends to infinity, with the retarded time $T_{R} \equiv T-R / c$ being fixed. In radiative coordinates the retarded time $T_{R}$ asymptotically coincides with a null coordinate. The waveform reads2

$$
h_{i j}^{\mathrm{TT}}=\frac{4 G}{c^{2} R} \mathcal{P}_{i j k l}^{\mathrm{TT}}(\mathbf{N}) \sum_{\ell=2}^{+\infty} \frac{N_{L-2}}{c^{\ell} \ell !}\left[U_{k l L-2}\left(T_{R}\right)-\frac{2 \ell}{c(\ell+1)} N_{m} \varepsilon_{m n(k} V_{l) n L-2}\left(T_{R}\right)\right]+\mathcal{O}\left(\frac{1}{R^{2}}\right)
$$

The waveform is parametrized by two sets of symmetric and trace-free (STF) multipole moments, $U_{L}$ of mass type and $V_{L}$ of current type, which constitute the observables of the gravitational wave at infinity and are called the radiative moments [61]. They are functions of the retarded time $T_{R}$ in the radiative coordinate system. Plugging Eq. (2.1) into the

\footnotetext{
${ }^{2}$ We denote by $L=i_{1} \cdots i_{\ell}$ a multi-index composed of $\ell$ multipolar spatial indices $i_{1}, \cdots, i_{\ell}$ ranging from 1 to 3 . Similarly $L-1=i_{1} \cdots i_{\ell-1}$ and $k L-2=k i_{1} \cdots i_{\ell-2} ; N_{L}=N_{i_{1}} \cdots N_{i_{\ell}}$ is the product of $\ell$ spatial vectors $N_{i}$. In the case of summed-up (dummy) multi-indices $L$, we do not write the $\ell$ summations from 1 to 3 over their indices. The transverse-traceless (TT) projection operator is denoted $\mathcal{P}_{i j k l}^{\mathrm{TT}}=\mathcal{P}_{i k} \mathcal{P}_{j l}-\frac{1}{2} \mathcal{P}_{i j} \mathcal{P}_{k l}$ where $\mathcal{P}_{i j}=\delta_{i j}-N_{i} N_{j}$ is the projector orthogonal to the unit direction $\mathbf{N}=\mathbf{X} / R$ of the radiative coordinate system $X^{\mu}=(c T, \mathbf{X})$. The quantity $\varepsilon_{i j k}$ is the Levi-Civita antisymmetric symbol such that $\varepsilon_{123}=1$. The symmetric-trace-free $(\mathrm{STF})$ projection is indicated using brackets or a hat. Thus $U_{L}=\hat{U}_{L}=U_{\langle L\rangle}$ and $V_{L}=\hat{V}_{L}=V_{\langle L\rangle}$ for STF moments. We denote time derivatives with a superscript $(n)$, and we indicate the symmetrization operation with round parentheses.
} 
standard expression for the gravitational-wave energy flux we get [61]

$$
\mathcal{F}=\sum_{\ell=2}^{+\infty} \frac{G}{c^{2 \ell+1}}\left[\frac{(\ell+1)(\ell+2)}{(\ell-1) \ell \ell !(2 \ell+1) ! !} U_{L}^{(1)} U_{L}^{(1)}+\frac{4 \ell(\ell+2)}{c^{2}(\ell-1)(\ell+1) !(2 \ell+1) ! !} V_{L}^{(1)} V_{L}^{(1)}\right]
$$

The radiative moments $U_{L}$ and $V_{L}$ are then related to some specific source-rooted multipole moments as follows. To implement the non-linearities in the propagation of the gravitational waves from the source to infinity, we express them as some non-linear functionals, which can in principle be developed at any order, of some "canonical" moments $M_{L}$ and $S_{L}$. When developped at the 1.5PN order they display the effect of tails and read [38, 41]

$$
\begin{aligned}
& U_{L}\left(T_{R}\right)=M_{L}^{(\ell)}+\frac{2 G M}{c^{3}} \int_{-\infty}^{T_{R}} \mathrm{~d} t M_{L}^{(\ell+2)}(t)\left[\ln \left(\frac{T_{R}-t}{2 \tau_{0}}\right)+\kappa_{\ell}\right]+\mathcal{O}\left(\frac{1}{c^{5}}\right), \\
& V_{L}\left(T_{R}\right)=S_{L}^{(\ell)}+\frac{2 G M}{c^{3}} \int_{-\infty}^{T_{R}} \mathrm{~d} t S_{L}^{(\ell+2)}(t)\left[\ln \left(\frac{T_{R}-t}{2 \tau_{0}}\right)+\pi_{\ell}\right]+\mathcal{O}\left(\frac{1}{c^{5}}\right)
\end{aligned}
$$

where $M$ is the mass monopole or Arnowitt-Deser-Misner (ADM) total mass. The quantities $\kappa_{\ell}$ and $\pi_{\ell}$ denote some numerical rational fractions and $\tau_{0}$ is an arbitrary constant time scale; we shall not need any of these here.

Next the canonical moments $M_{L}$ and $S_{L}$ themselves are given as some non-linear functionals of the "source" moments $I_{L}$ and $J_{L}$, and also of four supplementary "gauge" moments $W_{L}, X_{L}, Y_{L}$ and $Z_{L}$. In general, the canonical and source moments agree up to the 2.5PN order, namely

$$
\begin{aligned}
M_{L} & =I_{L}+\mathcal{O}\left(\frac{1}{c^{5}}\right), \\
S_{L} & =J_{L}+\mathcal{O}\left(\frac{1}{c^{5}}\right) .
\end{aligned}
$$

Since we address the computation of the spin-orbit 3.5PN contribution to the energy flux (2.2), we see that we only have to consider a possible spin-orbit $1 / c^{7}$ term in the mass quadrupole (since spin contributions add at least a factor $1 / c$ ). The relation between $M_{i j}$ and $I_{i j}$ is given by (see e.g. [62]):

$$
M_{i j}=I_{i j}+\frac{4 G}{c^{5}}\left[W^{(2)} I_{i j}-W^{(1)} I_{i j}^{(1)}\right]+\mathcal{O}\left(\frac{1}{c^{7}}\right) .
$$

As was already noticed in Ref. [33], the leading order spin contributions to $W$ and $I_{i j}$ both start at $\mathcal{O}\left(c^{-3}\right)$, so that $\left(M_{i j}\right)_{S}=\left(I_{i j}\right)_{S}+\mathcal{O}\left(c^{-8}\right)$. For our purposes, we can therefore ignore the distinction between canonical $M_{L}, S_{L}$ and source $I_{L}, J_{L}$ moments.

Finally the source multipole moments are defined for a general post-Newtonian matter source for any multipolar order $\ell \geq 2$, and up to any post-Newtonian order. They are explicitly given by $[43] 3$

$$
I_{L}(t)=\underset{B=0}{\mathrm{FP}} \int \mathrm{d}^{3} \mathbf{x}\left(r / r_{0}\right)^{B} \int_{-1}^{1} \mathrm{~d} z\left\{\delta_{\ell} \hat{x}_{L} \Sigma-\frac{4(2 \ell+1)}{c^{2}(\ell+1)(2 \ell+3)} \delta_{\ell+1} \hat{x}_{i L} \Sigma_{i}^{(1)}\right.
$$

\footnotetext{
${ }^{3}$ The brackets surrounding indices denote the STF projection; the STF product of $\ell$ spatial vectors is written as $\hat{x}_{L} \equiv x_{\left\langle i_{1}\right.} \cdots x_{\left.i_{\ell}\right\rangle} \equiv \operatorname{STF}\left[x_{L}\right]$.
} 


$$
\begin{gathered}
\left.+\frac{2(2 \ell+1)}{c^{4}(\ell+1)(\ell+2)(2 \ell+5)} \delta_{\ell+2} \hat{x}_{i j L} \Sigma_{i j}^{(2)}\right\}(\mathbf{x}, t+z r / c), \\
J_{L}(t)=\mathrm{FP}_{B=0} \varepsilon_{a b<i_{\ell}} \int \mathrm{d}^{3} \mathbf{x}\left(r / r_{0}\right)^{B} \int_{-1}^{1} \mathrm{~d} z\left\{\delta_{\ell} \hat{x}_{L-1>a} \Sigma_{b}\right. \\
\left.-\frac{2 \ell+1}{c^{2}(\ell+2)(2 \ell+3)} \delta_{\ell+1} \hat{x}_{L-1>a c} \Sigma_{b c}^{(1)}\right\}(\mathbf{x}, t+z r / c),
\end{gathered}
$$

The finite part operation FP in front represents an infra-red (IR) regularization defined by analytic continuation in a complex parameter $B$, and involves the same arbitrary scale $r_{0}=c \tau_{0}$ as in Eqs. (2.3), which will be irrelevant for the present work 4

The basic building "blocks" $\Sigma, \Sigma_{i}$ and $\Sigma_{i j}$ entering the latter formulas are evaluated at the position $\mathbf{x}$ and at time $t+z r / c$ in a harmonic coordinate system $(t, \mathbf{x})$ covering the source (where $r=|\mathbf{x}|$ ). They are defined by

$$
\Sigma \equiv \frac{\tau^{00}+\tau^{i i}}{c^{2}}, \quad \Sigma_{i} \equiv \frac{\tau^{0 i}}{c}, \quad \Sigma_{i j} \equiv \tau^{i j},
$$

together with $\tau^{i i} \equiv \delta_{i j} \tau^{i j}$. Here $\tau^{\mu \nu}$ denotes the post-Newtonian expansion of the total pseudo stress-energy tensor of the matter and gravitational fields, say

$$
\tau^{\mu \nu} \equiv \mathrm{PN}\left[|g| T^{\mu \nu}+\frac{c^{4}}{16 \pi G} \Lambda^{\mu \nu}(h)\right]
$$

where $T^{\mu \nu}$ is the stress-energy tensor of the matter source, and $\Lambda^{\mu \nu}(h)$ represents the gravitational source term which is given by a complicated non-linear, quadratic at least, functional of the field variable $h^{\mu \nu}$ and its first and second space-time derivatives. The pseudo-tensor appears in the right-hand-side of the Einstein field equations, when "relaxed" by the condition of harmonic (or de Donder) coordinates 5 The expressions (2.6) involve an intermediate integration over the variable $z$, with associated weighting function

$$
\delta_{\ell}(z) \equiv \frac{(2 \ell+1) ! !}{2^{\ell+1} \ell !}\left(1-z^{2}\right)^{\ell}, \quad \int_{-1}^{1} \mathrm{~d} z \delta_{\ell}(z)=1 .
$$

In practice the post-Newtonian expansion of the source moments (2.6) is performed by means of the formal infinite series

$$
\int_{-1}^{1} d z \delta_{\ell}(z) \Sigma(\mathbf{x}, t+z r / c)=\sum_{k=0}^{+\infty} \frac{(2 \ell+1) ! !}{(2 k) ! !(2 \ell+2 k+1) ! !}\left(\frac{r}{c}\right)^{2 k} \Sigma^{(2 k)}(\mathbf{x}, t) .
$$

\section{B. Explicit solution for the post-Newtonian metric}

To get explicit results at a given post-Newtonian order we need a solution of the relaxed Einstein field equations. As in Paper I, we parametrize an explicit solution by means of a

4 This scale enters the relation between the retarded time in radiative coordinates and the one in sourcerooted harmonic coordinates: $T_{R}=t-\frac{r}{c}-\frac{2 G M}{c^{3}} \ln \left(\frac{r}{r_{0}}\right)$.

5 The post-Newtonian expansion of the relaxed Einstein field equations takes the form $\square h^{\mu \nu}=\frac{16 \pi G}{c^{4}} \tau^{\mu \nu}$, where $\square \equiv \eta^{\rho \sigma} \partial_{\rho \sigma}$ is the flat space-time d'Alembertian operator. Here $h^{\mu \nu} \equiv \sqrt{-g} g^{\mu \nu}-\eta^{\mu \nu}$, where $g^{\mu \nu}$ is the inverse and $g$ the determinant of the usual covariant metric $g_{\mu \nu} ; \eta^{\mu \nu}$ is an auxiliary Minkowskian metric, $\eta^{\mu \nu}=\operatorname{diag}(-1,1,1,1)$. The harmonic-coordinate condition reads $\partial_{\nu} h^{\mu \nu}=0$. Note that the conservation of the pseudo tensor, $\partial_{\nu} \tau^{\mu \nu}=0$, is the consequence of the harmonic-coordinate condition. 
set of retarded potentials denoted by $V, V_{i}, \hat{W}_{i j}, \hat{R}_{i}, \hat{X}, \hat{Z}_{i j}$. Here, contrarily to our previous work on the equations of motion, we will not need the highest-order potentials $\hat{Y}_{i}$ and $\hat{T}$ that enter respectively $g_{0 i}$ at $\mathcal{O}\left(c^{-7}\right)$ and $g_{00}$ at $\mathcal{O}\left(c^{-8}\right)$. All these potentials are "Newtonian" in the sense that they admit a finite non-zero limit when $c \rightarrow+\infty$. They enter the components of the usual covariant metric as follows:

$$
\begin{aligned}
g_{00} & =-1+\frac{2}{c^{2}} V-\frac{2}{c^{4}} V^{2}+\frac{8}{c^{6}}\left(\hat{X}+V_{i} V_{i}+\frac{V^{3}}{6}\right)+\mathcal{O}\left(\frac{1}{c^{8}}\right), \\
g_{0 i} & =-\frac{4}{c^{3}} V_{i}-\frac{8}{c^{5}} \hat{R}_{i}+\mathcal{O}\left(\frac{1}{c^{7}}\right), \\
g_{i j} & =\delta_{i j}\left[1+\frac{2}{c^{2}} V+\frac{2}{c^{4}} V^{2}+\frac{8}{c^{6}}\left(\hat{X}+V_{k} V_{k}+\frac{V^{3}}{6}\right)\right] \\
& +\frac{4}{c^{4}} \hat{W}_{i j}+\frac{16}{c^{6}}\left(\hat{Z}_{i j}+\frac{1}{2} V \hat{W}_{i j}-V_{i} V_{j}\right)+\mathcal{O}\left(\frac{1}{c^{8}}\right) .
\end{aligned}
$$

Equivalently, and more useful for the present work, they enter the "gothic" metric as:

$$
\begin{aligned}
& \frac{h^{00}+h^{i i}}{2}=-\frac{2}{c^{2}} V-\frac{4}{c^{4}} V^{2}-\frac{8}{c^{6}}\left(\hat{X}+\frac{1}{2} V \hat{W}+\frac{2}{3} V^{3}\right)+\mathcal{O}\left(\frac{1}{c^{8}}\right), \\
& h^{0 i}=-\frac{4}{c^{3}} V_{i}-\frac{8}{c^{5}}\left(\hat{R}_{i}+V V_{i}\right)+\mathcal{O}\left(\frac{1}{c^{7}}\right), \\
& h^{i j}=-\frac{4}{c^{4}}\left(\hat{W}_{i j}-\frac{1}{2} \delta_{i j} \hat{W}\right)-\frac{16}{c^{6}}\left(\hat{Z}_{i j}-\frac{1}{2} \delta_{i j} \hat{Z}\right)+\mathcal{O}\left(\frac{1}{c^{8}}\right) .
\end{aligned}
$$

Each of these potentials is a retarded solution of a flat space-time wave equation sourced by matter densities components and appropriate lower order potentials. The matter densities are defined from the components of the matter stress-energy tensor by

$$
\sigma \equiv \frac{T^{00}+T^{i i}}{c^{2}}, \quad \sigma_{i} \equiv \frac{T^{0 i}}{c}, \quad \sigma_{i j} \equiv T^{i j}
$$

Denoting with $\square_{R}^{-1} S$ the usual retarded flat d'Alembertian integral, i.e. the retarded solution of $\square \phi \equiv \eta^{\mu \nu} \partial_{\mu \nu} \phi=S$, the latter potentials are defined by

$$
\begin{aligned}
& V= \square_{R}^{-1}[-4 \pi G \sigma], \\
& V_{i}=\square_{R}^{-1}\left[-4 \pi G \sigma_{i}\right], \\
& \hat{X}=\square_{R}^{-1}\left[-4 \pi G V \sigma_{i i}+\hat{W}_{i j} \partial_{i j} V+2 V_{i} \partial_{t} \partial_{i} V+V \partial_{t}^{2} V\right. \\
&\left.+\frac{3}{2}\left(\partial_{t} V\right)^{2}-2 \partial_{i} V_{j} \partial_{j} V_{i}\right], \\
& \hat{R}_{i}=\square_{R}^{-1} {\left[-4 \pi G\left(V \sigma_{i}-V_{i} \sigma\right)-2 \partial_{k} V \partial_{i} V_{k}-\frac{3}{2} \partial_{t} V \partial_{i} V\right], } \\
& \hat{W}_{i j}=\square_{R}^{-1}\left[-4 \pi G\left(\sigma_{i j}-\delta_{i j} \sigma_{k k}\right)-\partial_{i} V \partial_{j} V\right], \\
& \hat{Z}_{i j}=\square_{R}^{-1}\left[-4 \pi G V\left(\sigma_{i j}-\delta_{i j} \sigma_{k k}\right)-2 \partial_{(i} V \partial_{t} V_{j)}+\partial_{i} V_{k} \partial_{j} V_{k}+\partial_{k} V_{i} \partial_{k} V_{j}\right.
\end{aligned}
$$




$$
\left.-2 \partial_{(i} V_{k} \partial_{k} V_{j)}-\delta_{i j} \partial_{k} V_{m}\left(\partial_{k} V_{m}-\partial_{m} V_{k}\right)-\frac{3}{4} \delta_{i j}\left(\partial_{t} V\right)^{2}\right] .
$$

With the latter explicit post-Newtonian solution in hands one obtains the basic buiding blocks (2.7) entering the source multipole moments (2.6) as

$$
\begin{aligned}
& \Sigma=\left[1+\frac{4 V}{c^{2}}+\frac{4}{c^{4}}\left(2 V^{2}+\hat{W}\right)+\frac{16}{c^{6}}\left(V_{i} V_{i}+\frac{2}{3} V^{3}+V \hat{W}+\hat{X}+\hat{Z}\right)\right] \sigma-\frac{1}{\pi G c^{2}} \partial_{i} V \partial_{i} V \\
& +\frac{1}{\pi G c^{4}}\left\{-\frac{1}{2}\left(\partial_{t} V\right)^{2}-2 V_{i} \partial_{t} \partial_{i} V-V \partial_{t}^{2} V-\frac{7}{2} V \partial_{i} V \partial_{i} V-\hat{W}_{i j} \partial_{i j}^{2} V-\partial_{i} \hat{W} \partial_{i} V\right. \\
& \left.+2 \partial_{i} V_{j} \partial_{j} V_{i}+\frac{1}{2} \hat{W} \partial_{i i} V\right\} \\
& +\frac{1}{\pi G c^{6}}\left\{-\partial_{t} V \partial_{t} \hat{W}-\frac{7}{2}\left(\partial_{t} V\right)^{2} V+2 \partial_{t} V_{i} \partial_{t} V_{i}-4 \hat{R}_{i} \partial_{t} \partial_{i} V-12 V V_{i} \partial_{t} \partial_{i} V\right. \\
& -6 V^{2} \partial_{t}^{2} V-\frac{1}{2} \hat{W} \partial_{t}^{2} V-6 V_{i} \partial_{t} V \partial_{i} V+4 V \partial_{t} V_{i} \partial_{i} V-7 V^{2} \partial_{i} V \partial_{i} V \\
& -8 \partial_{i} \hat{X} \partial_{i} V-\hat{W}_{i j} \partial_{i} V \partial_{j} V-2 \partial_{t} \hat{W} \partial_{i} V_{i}-4 V \hat{W}_{i j} \partial_{i j} V-4 \hat{Z}_{i j} \partial_{i j} V \\
& +8 \partial_{i} V_{j} \partial_{j} \hat{R}_{i}-\frac{3}{2} \hat{W} \partial_{i} V \partial_{i} V-8 V_{i} \partial_{j} V_{i} \partial_{j} V-4 V \partial_{i} \hat{W} \partial_{i} V-4 \partial_{i} \hat{Z} \partial_{i} V \\
& +4 \partial_{t} \hat{W}_{i j} \partial_{j} V_{i}+6 V \partial_{i} V_{j} \partial_{j} V_{i}+2 V \partial_{j} V_{i} \partial_{j} V_{i}+2 V \hat{W} \partial_{i i} V+2 \hat{Z} \partial_{i i} V \\
& \left.+\partial_{i} \hat{W}_{j k} \partial_{i} \hat{W}_{j k}-\frac{1}{2} \partial_{i} \hat{W} \partial_{i} \hat{W}\right\}+\mathcal{O}\left(\frac{1}{c^{8}}\right), \\
& \Sigma_{i}=\left[1+\frac{4 V}{c^{2}}+\frac{4}{c^{4}}\left(2 V^{2}+\hat{W}\right)\right] \sigma_{i}+\frac{1}{\pi G c^{2}}\left\{\partial_{k} V\left(\partial_{i} V_{k}-\partial_{k} V_{i}\right)+\frac{3}{4} \partial_{t} V \partial_{i} V\right\} \\
& +\frac{1}{\pi G c^{4}}\left\{\partial_{t} V \partial_{t} V_{i}-2 V_{j} \partial_{t} \partial_{j} V_{i}-V \partial_{t}^{2} V_{i}-2 \partial_{j} V \partial_{j} \hat{R}_{i}+\partial_{t} \hat{W}_{i j} \partial_{j} V-\frac{3}{2} V_{i} \partial_{j} V \partial_{j} V\right. \\
& -2 V \partial_{j} V_{i} \partial_{j} V-\hat{W}_{j k} \partial_{j k} V_{i}+\partial_{j} \hat{W}_{i k} \partial_{k} V_{j}+\partial_{k} \hat{W}_{i j} \partial_{k} V_{j}-\partial_{k} \hat{W} \partial_{k} V_{i} \\
& +2 \partial_{j} V \partial_{i} \hat{R}_{j}+3 V \partial_{t} V \partial_{i} V+V_{j} \partial_{j} V \partial_{i} V+2 V \partial_{j} V \partial_{i} V_{j}-\partial_{k} V_{j} \partial_{i} \hat{W}_{j k} \\
& \left.+\frac{1}{2} \partial_{t} V \partial_{i} \hat{W}+\frac{1}{2} \partial_{j} V_{j} \partial_{i} \hat{W}+\frac{1}{2} \hat{W} \partial_{j j} V_{i}\right\}+\mathcal{O}\left(\frac{1}{c^{6}}\right), \\
& \Sigma_{i j}=\left[1+\frac{4 V}{c^{2}}\right] \sigma_{i j}+\frac{1}{\pi G}\left\{-\frac{1}{8} \delta_{i j} \partial_{k} V \partial_{k} V+\frac{1}{4} \partial_{i} V \partial_{j} V\right\} \\
& +\frac{1}{\pi G c^{2}}\left\{\left(-\frac{3}{8}\left(\partial_{t} V\right)^{2}-\partial_{t} V_{k} \partial_{k} V-\frac{1}{2} \partial_{k} V_{l} \partial_{l} V_{k}+\frac{1}{2} \partial_{k} V_{l} \partial_{k} V_{l}\right) \delta_{i j}-\partial_{k} V_{i} \partial_{k} V_{j}\right. \\
& \left.+\partial_{t} V_{j} \partial_{i} V+\partial_{k} V_{j} \partial_{i} V_{k}+\partial_{t} V_{i} \partial_{j} V+\partial_{k} V_{i} \partial_{j} V_{k}-\partial_{i} V_{k} \partial_{j} V_{k}\right\}+\mathcal{O}\left(\frac{1}{c^{4}}\right),
\end{aligned}
$$

where we have used the notation $\hat{W}=\hat{W}_{i i}$ and $\hat{Z}=\hat{Z}_{i i}$. Equivalent expressions for $\Sigma, \Sigma_{i}$ and $\Sigma_{i j}$, which we have used for testing our calculations, are provided in Appendix $\mathrm{A}$. 


\section{APPLICATION TO SPINNING COMPACT BINARIES}

\section{A. The pole-dipole effective formalism}

The pole-dipole formalism [45 55] is an effective description of point particles endowed with intrinsic (classical) angular momenta or spins, and moving in an arbitrary curved background - in practice the space-time generated by the particles themselves. The spins can take any orientation and magnitude, and in particular be close to extremal. In the present work we shall confine the formalism to terms linear in the spins. At that level the model can be used for describing black holes as well as ordinary compact bodies like neutrons stars. Indeed, the internal structure of the spinning bodies should appear only at the quadratic level in the spins, e.g. through the rotationally induced quadrupole moment.

The stress-energy tensor of each of the particles is the sum of two terms, respectively built with a Dirac delta function and a gradient of a delta function, and integrated over the world line of the particle, according to:

$$
T^{\mu \nu}(x)=\sum_{1,2} c^{2} \int_{-\infty}^{+\infty} \mathrm{d} \tau p^{(\mu} u^{\nu)} \frac{\delta^{(4)}(x-y(\tau))}{\sqrt{-g(x)}}-c \int_{-\infty}^{+\infty} \mathrm{d} \tau \nabla_{\rho}\left[S^{\rho(\mu} u^{\nu)} \frac{\delta^{(4)}(x-y(\tau))}{\sqrt{-g(x)}}\right] .
$$

Here the sum is over the two particles, $\tau$ is the proper time measured along the world line of each particle given by the particle position $y^{\mu}(\tau) ; \delta^{(4)}$ denotes the four-dimensional Dirac delta function; $u^{\mu}=\mathrm{d} y^{\mu} /(c \mathrm{~d} \tau)$ is the four velocity of the particle normalized to $u_{\mu} u^{\mu}=-1 ; p^{\mu}$ is its four linear momentum; and $S^{\mu \nu}$ denotes the antisymmetric tensor that represents the spin of the particle 6 Using a $3+1$ space-time split, the particle's position and coordinate velocity are denoted $y^{\mu}=(c t, \mathbf{y}(t))$ and $v^{\mu}(t)=(c, \mathbf{v}(t))$ (where $v^{\mu}=c u^{\mu} / u^{0}$, with $\left.u^{0}=\left[-g_{\rho \sigma} v^{\rho} v^{\sigma} / c^{2}\right]^{-1 / 2}\right)$, and we have

$$
T^{\mu \nu}(\mathbf{x}, t)=\sum_{1,2} p^{(\mu} v^{\nu)} \frac{\delta^{(3)}(\mathbf{x}-\mathbf{y}(t))}{\sqrt{-g(\mathbf{x}, t)}}-\frac{1}{c} \nabla_{\rho}\left[S^{\rho(\mu} v^{\nu)} \frac{\delta^{(3)}(\mathbf{x}-\mathbf{y}(t))}{\sqrt{-g(\mathbf{x}, t)}}\right]
$$

where $\delta^{(3)}$ is the three-dimensional Dirac delta function and the spin tensor $S^{\mu \nu}(t)$ is considered a function of coordinate time.

Since the spin tensor $S^{\mu \nu}$ has six independent components, one must impose a supplementary spin condition (SSC) in order to correctly describe the three independent components of the spin vector. Here we adopt the covariant SSC (Tulczyjew's condition [49, 50])

$$
S^{\mu \nu} p_{\nu}=0 \text {. }
$$

It can be shown that, with the latter choice for the SSC, the mass defined by $m^{2} c^{2}=-p^{\mu} p_{\mu}$ as well as the (four-dimensional) magnitude of the spin defined by $S^{2}=S^{\mu \nu} S_{\mu \nu} / 2$, are conserved along the particle's trajectory. Furthermore the link between the four velocity $u^{\mu}$ and the four linear momentum $p^{\mu}$ is entirely specified. However, at linear order in the spins the linear momentum is simply proportional to the four velocity,

$$
p^{\mu}=m c u^{\mu}+\mathcal{O}\left(S^{2}\right),
$$

\footnotetext{
${ }^{6}$ In our convention the spin tensor and all spin variables have the dimension of an angular momentum times the speed of light $c$. This is useful for counting the effects of spins in the post-Newtonian approximation, as the spins appear to be formally "Newtonian" for maximally spinning particles. All the powers of $1 / c$ are kept explicitly in our calculations, so that the next-to-next-to-leading spin-orbit terms we are looking for here will all carry in front a factor $1 / c^{7}$.
} 
so that the covariant SSC reduces to $S^{\mu \nu} u_{\nu}=\mathcal{O}\left(S^{3}\right)$. Furthermore it can be shown that the spin precession equation reduces to the parallel transport equation

$$
\frac{\mathrm{D} S^{\mu \nu}}{\mathrm{d} \tau}=\mathcal{O}\left(S^{2}\right)
$$

where $\mathrm{D} / \mathrm{d} \tau$ is the proper time covariant derivative, while the four dimensional acceleration of the particle is given by

$$
\frac{\mathrm{D} u^{\mu}}{\mathrm{d} \tau}=-\frac{1}{2 m c} R_{\nu \rho \sigma}^{\mu} u^{\nu} S^{\rho \sigma}+\mathcal{O}\left(S^{2}\right)
$$

This equation of motion is well known as the Mathisson-Papapetrou equation [45 48].

\section{B. Multipole moments in the center-of-mass frame}

We work in all intermediate calculations with the spatial components $S_{1}^{i j}$ and $S_{2}^{i j}$ of the two spin tensors $(i, j=1,2,3)$, eliminating the $0 i$ components thanks to the covariant SSC (3.3), and then express all our results in terms of some spin vectors $S_{1}^{i}$ and $S_{2}^{i}$. Like in Paper II we shall adopt a spin vector whose magnitude is constant in the usual three-dimensional Euclidean sense, namely

$$
\delta_{i j} S_{1}^{i} S_{1}^{j}=s_{1}^{2}, \quad \delta_{i j} S_{2}^{i} S_{2}^{j}=s_{2}^{2}, \quad s_{1,2}=\text { const } .
$$

We refer to Section IIA in Paper II for the precise construction, starting from the spatial components of the spin tensors, of spin vector variables $S_{1}^{i}$ and $S_{2}^{i}$ with constant magnitude, Eq. (3.7). Such spin vectors therefore satisfy ordinary-looking precession equations,

$$
\frac{\mathrm{d} S_{1}^{i}}{\mathrm{~d} t}=\varepsilon^{i j k} \Omega_{1}^{j} S_{1}^{k}, \quad \frac{\mathrm{d} S_{2}^{i}}{\mathrm{~d} t}=\varepsilon^{i j k} \Omega_{2}^{j} S_{2}^{k} .
$$

All the problem of the evolution of the spins $S_{1}^{i}$ and $S_{2}^{i}$ in a binary system reduces to that of finding the ordinary precession vectors $\Omega_{1}^{i}$ and $\Omega_{2}^{i}$. Those have been obtained for the two particles up to 3PN order in Section IIB of Paper II (for spin-orbit effects the precession vectors are independent of the spins). Now that we have defined the two constant magnitude spins $S_{1}^{i}$ and $S_{2}^{i}$ it will be convenient to express the results in the center-of-mass frame by means of the variables [17]

$$
S^{i} \equiv S_{1}^{i}+S_{2}^{i}, \quad \Sigma^{i} \equiv \frac{m}{m_{2}} S_{2}^{i}-\frac{m}{m_{1}} S_{1}^{i}
$$

Here we denote the two individual masses by $m_{1}$ and $m_{2}$, the total mass by $m \equiv m_{1}+m_{2}$, and later we shall use the symmetric mass ratio $\nu \equiv m_{1} m_{2} / m^{2}($ such that $0<\nu \leqslant 1 / 4)$, and the mass difference $\delta m \equiv m_{1}-m_{2}$.

We compute the spin-orbit terms in the various source multipole moments which will enter the flux up to 3.5PN order in an arbitrary frame. At this stage, an interesting check described in Appendix B can be performed. Next, we adopt the frame of the center-of-mass (CM) defined in Paper II; notably the particle's trajectories $y_{1}^{i}$ and $y_{2}^{i}$ in the CM frame are given in terms of the relative position $x^{i} \equiv y_{1}^{i}-y_{2}^{i}$ and relative velocity $v^{i} \equiv \mathrm{d} x^{i} / \mathrm{d} t=v_{1}^{i}-v_{2}^{i}$ by Eqs. (3.3) in Paper II. Finally the spin parts of the multipole moments are expressed 
in terms of the conserved-magnitude spins and of the variables (3.9). Our notation is e.g. $(v S) \equiv \mathbf{v} \cdot \mathbf{S}$ for the ordinary Euclidean scalar product, e.g. $(\mathbf{x} \times \mathbf{\Sigma})^{i} \equiv \varepsilon^{i j k} x^{j} \Sigma^{k}$ for the ordinary cross product, and $\operatorname{e.g} .(S, x, v) \equiv \mathbf{S} \cdot(\mathbf{x} \times \mathbf{v})=\varepsilon^{i j k} S^{i} x^{j} v^{k}$ for the mixed product. For each source multipole moment, we only present the linear-in-spin part, indicated by the underneath label $S$. We obtain, extending Ref. [21] to next-to-next-to-leading order and writing all terms using our conserved spin variables,

$$
\begin{aligned}
& { }_{S}^{I} i j=\frac{r \nu}{c^{3}}\left\{-\frac{8}{3}(\mathbf{S} \times \mathbf{v})^{<i} n^{j>}-\frac{8}{3} \frac{\delta m}{m}(\mathbf{\Sigma} \times \mathbf{v})^{<i} n^{j>}\right. \\
& \left.-\frac{4}{3}(\mathbf{n} \times \mathbf{S})^{<i} v^{j>}-\frac{4}{3} \frac{\delta m}{m}(\mathbf{n} \times \mathbf{\Sigma})^{<i} v^{j>}\right\} \\
& +\frac{r \nu}{c^{5}}\left[\left\{(\mathbf{S} \times \mathbf{v})^{<i} n^{j>}\left(-\frac{26}{21}+\frac{26}{7} \nu\right) v^{2}+(\boldsymbol{\Sigma} \times \mathbf{v})^{<i} n^{j>} \frac{\delta m}{m}\left(-\frac{26}{21}+\frac{116}{21} \nu\right) v^{2}\right.\right. \\
& +(\mathbf{n} \times \mathbf{S})^{<i} v^{j>}\left(-\frac{4}{21}+\frac{4}{7} \nu\right) v^{2}+(\mathbf{n} \times \boldsymbol{\Sigma})^{<i} v^{j>} \frac{\delta m}{m}\left(-\frac{4}{21}+\frac{12}{7} \nu\right) v^{2} \\
& +(\mathbf{S} \times \mathbf{v})^{<i} v^{j>}\left(\frac{4}{21}-\frac{4}{7} \nu\right)(n v)+(\boldsymbol{\Sigma} \times \mathbf{v})^{<i} v^{j>} \frac{\delta m}{m}\left(\frac{4}{21}-\frac{20}{21} \nu\right)(n v) \\
& \left.+(n, S, v) v^{<i} v^{j>}\left(-\frac{3}{7}+\frac{9}{7} \nu\right)+(n, \Sigma, v) v^{<i} v^{j>} \frac{\delta m}{m}\left(-\frac{3}{7}+\frac{40}{21} \nu\right)\right\} \\
& +\frac{G m}{r}\left\{(n, S, v) n^{<i} n^{j>}\left(-\frac{38}{21}-\frac{4}{7} \nu\right)+(n, \Sigma, v) n^{<i} n^{j>} \frac{\delta m}{m}\left(-\frac{16}{7}+\frac{26}{21} \nu\right)\right. \\
& +(\mathbf{n} \times \mathbf{S})^{<i} n^{j>}\left(\frac{17}{21}+\frac{61}{21} \nu\right)(n v)+(\mathbf{n} \times \boldsymbol{\Sigma})^{<i} n^{j>} \frac{\delta m}{m}\left(1+\frac{34}{21} \nu\right)(n v) \\
& +(n S)(\mathbf{n} \times \mathbf{v})^{<i} n^{j>}\left(-2+\frac{10}{3} \nu\right)+(n \Sigma)(\mathbf{n} \times \mathbf{v})^{<i} n^{j>} \frac{\delta m}{m}\left(-2+\frac{4}{3} \nu\right) \\
& +(\mathbf{S} \times \mathbf{v})^{<i} n^{j>}\left(-\frac{11}{7}-\frac{125}{21} \nu\right)+(\mathbf{\Sigma} \times \mathbf{v})^{<i} n^{j>} \frac{\delta m}{m}\left(-\frac{1}{3}-\frac{16}{3} \nu\right) \\
& \left.\left.+(\mathbf{n} \times \mathbf{S})^{<i} v^{j>}\left(-\frac{22}{3}-\frac{10}{3} \nu\right)+(\mathbf{n} \times \mathbf{\Sigma})^{<i} v^{j>} \frac{\delta m}{m}\left(-\frac{8}{3}-\frac{34}{21} \nu\right)\right\}\right] \\
& +\frac{r \nu}{c^{7}}\left[\left\{(\mathbf{S} \times \mathbf{v})^{<i} n^{j>}\left(-\frac{58}{63}+\frac{404}{63} \nu-\frac{746}{63} \nu^{2}\right) v^{4}\right.\right. \\
& +(\boldsymbol{\Sigma} \times \mathbf{v})^{<i} n^{j>} \frac{\delta m}{m}\left(-\frac{58}{63}+\frac{542}{63} \nu-\frac{1262}{63} \nu^{2}\right) v^{4} \\
& +(\mathbf{n} \times \mathbf{S})^{<i} v^{j>}\left(-\frac{4}{63}+\frac{23}{63} \nu-\frac{32}{63} \nu^{2}\right) v^{4} \\
& +(\mathbf{n} \times \boldsymbol{\Sigma})^{<i} v^{j>} \frac{\delta m}{m}\left(-\frac{4}{63}+\frac{103}{63} \nu-\frac{16}{3} \nu^{2}\right) v^{4} \\
& +(\mathbf{S} \times \mathbf{v})^{<i} v^{j>}\left(\frac{25}{126}-\frac{173}{126} \nu+\frac{317}{126} \nu^{2}\right)(n v) v^{2} \\
& +(\boldsymbol{\Sigma} \times \mathbf{v})^{<i} v^{j>} \frac{\delta m}{m}\left(\frac{25}{126}-\frac{118}{63} \nu+\frac{575}{126} \nu^{2}\right)(n v) v^{2}
\end{aligned}
$$




$$
\begin{aligned}
& +(n, S, v) v^{<i} v^{j>}\left(-\frac{25}{63}+\frac{179}{63} \nu-\frac{341}{63} \nu^{2}\right) v^{2} \\
& \left.+(n, \Sigma, v) v^{<i} v^{j>} \frac{\delta m}{m}\left(-\frac{25}{63}+\frac{76}{21} \nu-\frac{550}{63} \nu^{2}\right) v^{2}\right\} \\
& +\frac{G m}{r}\left\{(n, S, v) n^{<i} n^{j>}\left[\left(-\frac{148}{63}+\frac{542}{63} \nu+\frac{625}{63} \nu^{2}\right)(n v)^{2}+\left(\frac{586}{189}-\frac{2354}{189} \nu-\frac{208}{189} \nu^{2}\right) v^{2}\right]\right. \\
& +(n, \Sigma, v) n^{<i} n^{j>}\left[\frac{\delta m}{m}\left(-\frac{167}{126}+\frac{53}{14} \nu+\frac{949}{126} \nu^{2}\right)(n v)^{2}+\frac{\delta m}{m}\left(-\frac{19}{378}+\frac{27}{14} \nu-\frac{4231}{378} \nu^{2}\right) v^{2}\right] \\
& +(\mathbf{n} \times \mathbf{S})^{<i} n^{j>}\left[\left(\frac{499}{252}-\frac{1451}{252} \nu-\frac{3487}{252} \nu^{2}\right)(n v)^{3}+\left(\frac{85}{252}+\frac{1369}{252} \nu-\frac{3643}{252} \nu^{2}\right)(n v) v^{2}\right] \\
& +(\mathbf{n} \times \boldsymbol{\Sigma})^{<i} n^{j>}\left[\frac{\delta m}{m}\left(\frac{253}{252}-\frac{220}{63} \nu-\frac{187}{28} \nu^{2}\right)(n v)^{3}+\frac{\delta m}{m}\left(\frac{89}{84}+\frac{11}{14} \nu-\frac{715}{84} \nu^{2}\right)(n v) v^{2}\right] \\
& +(n S)(\mathbf{n} \times \mathbf{v})^{<i} n^{j>}\left[\left(-\frac{9}{14}+\frac{33}{14} \nu+\frac{89}{14} \nu^{2}\right)(n v)^{2}+\left(\frac{5}{42}+\frac{5}{42} \nu-\frac{415}{42} \nu^{2}\right) v^{2}\right] \\
& +(n \Sigma)(\mathbf{n} \times \mathbf{v})^{<i} n^{j>}\left[\frac{\delta m}{m}\left(-\frac{9}{14}+\frac{12}{7} \nu+\frac{39}{14} \nu^{2}\right)(n v)^{2}+\frac{\delta m}{m}\left(\frac{5}{42}+\frac{5}{21} \nu-\frac{57}{14} \nu^{2}\right) v^{2}\right] \\
& +(S v)(\mathbf{n} \times \mathbf{v})^{<i} n^{j>}\left(-\frac{10}{7}+\frac{110}{21} \nu-\frac{10}{7} \nu^{2}\right)(n v) \\
& +(\Sigma v)(\mathbf{n} \times \mathbf{v})^{<i} n^{j>} \frac{\delta m}{m}\left(-\frac{10}{7}+\frac{80}{21} \nu-\frac{10}{21} \nu^{2}\right)(n v) \\
& +(\mathbf{S} \times \mathbf{v})^{<i} n^{j>}\left[\left(\frac{2705}{756}-\frac{991}{108} \nu-\frac{9077}{756} \nu^{2}\right)(n v)^{2}+\left(-\frac{755}{108}+\frac{7507}{756} \nu+\frac{13655}{756} \nu^{2}\right) v^{2}\right] \\
& +(\boldsymbol{\Sigma} \times \mathbf{v})^{<i} n^{j>}\left[\frac{\delta m}{m}\left(\frac{2117}{756}-\frac{14}{3} \nu-\frac{691}{756} \nu^{2}\right)(n v)^{2}+\frac{\delta m}{m}\left(-\frac{4637}{756}+\frac{1993}{126} \nu+\frac{17419}{756} \nu^{2}\right) v^{2}\right] \\
& +(n, S, v) n^{<i} v^{j>}\left(-\frac{284}{189}+\frac{2203}{189} \nu-\frac{3784}{189} \nu^{2}\right)(n v) \\
& +(n, \Sigma, v) n^{<i} v^{j>} \frac{\delta m}{m}\left(-\frac{125}{189}+\frac{34}{9} \nu-\frac{410}{189} \nu^{2}\right)(n v) \\
& +(\mathbf{n} \times \mathbf{S})^{<i} v^{j>}\left[\left(-\frac{95}{126}-\frac{407}{126} \nu+\frac{4175}{126} \nu^{2}\right)(n v)^{2}+\left(-\frac{25}{7}+\frac{14}{3} \nu+\frac{128}{21} \nu^{2}\right) v^{2}\right] \\
& +(\mathbf{n} \times \boldsymbol{\Sigma})^{<i} v^{j>}\left[\frac{\delta m}{m}\left(-\frac{13}{126}+\frac{122}{63} \nu+\frac{127}{6} \nu^{2}\right)(n v)^{2}+\frac{\delta m}{m}\left(-\frac{121}{63}+\frac{482}{63} \nu+\frac{274}{63} \nu^{2}\right) v^{2}\right] \\
& +(n S)(\mathbf{n} \times \mathbf{v})^{<i} v^{j>}\left(\frac{3}{14}-\frac{11}{14} \nu+\frac{3}{14} \nu^{2}\right)(n v) \\
& +(n \Sigma)(\mathbf{n} \times \mathbf{v})^{<i} v^{j>} \frac{\delta m}{m}\left(\frac{3}{14}-\frac{4}{7} \nu+\frac{1}{14} \nu^{2}\right)(n v) \\
& +(S v)(\mathbf{n} \times \mathbf{v})^{<i} v^{j>}\left(\frac{5}{7}-\frac{55}{21} \nu+\frac{5}{7} \nu^{2}\right) \\
& +(\Sigma v)(\mathbf{n} \times \mathbf{v})^{<i} v^{j>} \frac{\delta m}{m}\left(\frac{5}{7}-\frac{40}{21} \nu+\frac{5}{21} \nu^{2}\right)
\end{aligned}
$$




$$
\begin{aligned}
& +(\mathbf{S} \times \mathbf{v})^{<i} v^{j>}\left(-\frac{191}{378}-\frac{1511}{378} \nu+\frac{1133}{54} \nu^{2}\right)(n v) \\
& +(\boldsymbol{\Sigma} \times \mathbf{v})^{<i} v^{j>} \frac{\delta m}{m}\left(-\frac{95}{378}-\frac{262}{63} \nu+\frac{415}{54} \nu^{2}\right)(n v) \\
& +(n, S, v) v^{<i} v^{j>}\left(-\frac{326}{189}+\frac{55}{189} \nu+\frac{389}{189} \nu^{2}\right) \\
& \left.+(n, \Sigma, v) v^{<i} v^{j>} \frac{\delta m}{m}\left(-\frac{506}{189}+\frac{59}{9} \nu+\frac{319}{189} \nu^{2}\right)\right\} \\
& +\frac{G^{2} m^{2}}{r^{2}}\left\{(n, S, v) n^{<i} n^{j>}\left(-\frac{2543}{252}-\frac{18083}{252} \nu+\frac{1319}{252} \nu^{2}\right)\right. \\
& +(n, \Sigma, v) n^{<i} n^{j>} \frac{\delta m}{m}\left(-\frac{563}{108}-\frac{2951}{84} \nu+\frac{805}{108} \nu^{2}\right) \\
& +(\mathbf{n} \times \mathbf{S})^{<i} n^{j>}\left(\frac{937}{84}+\frac{12497}{252} \nu+\frac{2039}{252} \nu^{2}\right)(n v) \\
& +(\mathbf{n} \times \boldsymbol{\Sigma})^{<i} n^{j>} \frac{\delta m}{m}\left(\frac{3289}{756}+\frac{21145}{756} \nu+\frac{4289}{756} \nu^{2}\right)(n v) \\
& +(n S)(\mathbf{n} \times \mathbf{v})^{<i} n^{j>}\left(-\frac{1}{3}+\frac{4}{3} \nu+\frac{13}{3} \nu^{2}\right) \\
& +(n \Sigma)(\mathbf{n} \times \mathbf{v})^{<i} n^{j>} \frac{\delta m}{m}\left(-\frac{1}{3}-\frac{5}{3} \nu+\frac{4}{3} \nu^{2}\right) \\
& +(\mathbf{S} \times \mathbf{v})^{<i} n^{j>}\left(\frac{559}{252}+\frac{17693}{252} \nu-\frac{647}{84} \nu^{2}\right) \\
& +(\boldsymbol{\Sigma} \times \mathbf{v})^{<i} n^{j>} \frac{\delta m}{m}\left(\frac{4681}{756}+\frac{38987}{756} \nu-\frac{1781}{252} \nu^{2}\right) \\
& +(\mathbf{n} \times \mathbf{S})^{<i} v^{j>}\left(-\frac{311}{63}+\frac{56}{3} \nu+\frac{13}{63} \nu^{2}\right) \\
& \left.\left.+(\mathbf{n} \times \boldsymbol{\Sigma})^{<i} v^{j>} \frac{\delta m}{m}\left(\frac{100}{189}+\frac{3211}{189} \nu+\frac{482}{189} \nu^{2}\right)\right\}\right] \\
& +\mathcal{O}\left(\frac{1}{c^{9}}\right) \\
& \begin{aligned}
J_{S} i j=\frac{r \nu}{c}\{- & \left.\frac{3}{2} \Sigma^{<i} n^{j>}\right\} \\
+\frac{r \nu}{c^{3}}[\{- & \frac{2}{7} \frac{\delta m}{m} v^{2} S^{<i} n^{j>}+\Sigma^{<i} n^{j>}\left(-\frac{29}{28}+\frac{143}{28} \nu\right) v^{2} \\
& +\frac{33}{28} \frac{\delta m}{m}(S v) n^{<i} v^{j>}+(\Sigma v) n^{<i} v^{j>}\left(\frac{33}{28}-\frac{155}{28} \nu\right) \\
& +\frac{3}{7} \frac{\delta m}{m}(n v) S^{<i} v^{j>}+\Sigma^{<i} v^{j>}\left(\frac{3}{7}-\frac{16}{7} \nu\right)(n v)
\end{aligned}
\end{aligned}
$$




$$
\begin{aligned}
& \left.-\frac{11}{14} \frac{\delta m}{m}(n S) v^{<i} v^{j>}+(n \Sigma) v^{<i} v^{j>}\left(-\frac{11}{14}+\frac{47}{14} \nu\right)\right\} \\
& +\frac{G m}{r}\left\{-\frac{29}{14} \frac{\delta m}{m}(n S) n^{<i} n^{j>}+(n \Sigma) n^{<i} n^{j>}\left(-\frac{4}{7}+\frac{31}{14} \nu\right)\right. \\
& \left.\left.+\frac{10}{7} \frac{\delta m}{m} S^{<i} n^{j>}+\Sigma^{<i} n^{j>}\left(\frac{61}{28}-\frac{71}{28} \nu\right)\right\}\right] \\
& +\frac{r \nu}{c^{5}}\left[\left\{S^{<i} n^{j>} \frac{\delta m}{m}\left(-\frac{4}{21}+\frac{17}{21} \nu\right) v^{4}+\Sigma^{<i} n^{j>}\left(-\frac{253}{336}+\frac{2435}{336} \nu-\frac{5633}{336} \nu^{2}\right) v^{4}\right.\right. \\
& +(S v) n^{<i} v^{j>} \frac{\delta m}{m}\left(\frac{269}{336}-\frac{283}{84} \nu\right) v^{2}+(\Sigma v) n^{<i} v^{j>}\left(\frac{269}{336}-\frac{2587}{336} \nu+\frac{6001}{336} \nu^{2}\right) v^{2} \\
& +S^{<i} v^{j>} \frac{\delta m}{m}\left(\frac{13}{42}-\frac{53}{42} \nu\right)(n v) v^{2}+\Sigma^{<i} v^{j>}\left(\frac{13}{42}-\frac{125}{42} \nu+\frac{293}{42} \nu^{2}\right)(n v) v^{2} \\
& +(n S) v^{<i} v^{j>} \frac{\delta m}{m}\left(-\frac{41}{84}+\frac{181}{84} \nu\right) v^{2}+(n \Sigma) v^{<i} v^{j>}\left(-\frac{41}{84}+\frac{55}{12} \nu-\frac{443}{42} \nu^{2}\right) v^{2} \\
& \left.+(S v) v^{<i} v^{j>} \frac{\delta m}{m}\left(\frac{5}{84}-\frac{5}{42} \nu\right)(n v)+(\Sigma v) v^{<i} v^{j>}\left(\frac{5}{84}-\frac{10}{21} \nu+\frac{5}{6} \nu^{2}\right)(n v)\right\} \\
& +\frac{G m}{r}\left\{(n S) n^{<i} n^{j>}\left[\frac{\delta m}{m}\left(\frac{23}{168}+\frac{415}{84} \nu\right)(n v)^{2}+\frac{\delta m}{m}\left(-\frac{617}{504}+\frac{809}{252} \nu\right) v^{2}\right]\right. \\
& +(n \Sigma) n^{<i} n^{j>}\left[\left(-\frac{13}{168}+\frac{569}{168} \nu-\frac{2147}{168} \nu^{2}\right)(n v)^{2}+\left(\frac{229}{504}-\frac{1703}{504} \nu+\frac{2795}{504} \nu^{2}\right) v^{2}\right] \\
& +(S v) n^{<i} n^{j>} \frac{\delta m}{m}\left(\frac{331}{252}+\frac{2549}{504} \nu\right)(n v) \\
& +(\Sigma v) n^{<i} n^{j>}\left(-\frac{101}{252}+\frac{449}{72} \nu-\frac{2789}{126} \nu^{2}\right)(n v) \\
& +S^{<i} n^{j>}\left[\frac{\delta m}{m}\left(-\frac{115}{126}-\frac{487}{126} \nu\right)(n v)^{2}+\frac{\delta m}{m}\left(\frac{125}{63}-\frac{157}{63} \nu\right) v^{2}\right] \\
& +\Sigma^{<i} n^{j>}\left[\left(\frac{163}{144}-\frac{10529}{1008} \nu+\frac{25247}{1008} \nu^{2}\right)(n v)^{2}+\left(-\frac{3175}{1008}+\frac{18413}{1008} \nu+\frac{7009}{1008} \nu^{2}\right) v^{2}\right] \\
& +(n S) n^{<i} v^{j>} \frac{\delta m}{m}\left(-\frac{317}{126}-\frac{497}{72} \nu\right)(n v) \\
& +(n \Sigma) n^{<i} v^{j>}\left(-\frac{173}{126}+\frac{2389}{504} \nu+\frac{1073}{126} \nu^{2}\right)(n v) \\
& +(S v) n^{<i} v^{j>} \frac{\delta m}{m}\left(\frac{5}{504}+\frac{1649}{504} \nu\right) \\
& +(\Sigma v) n^{<i} v^{j>}\left(\frac{2021}{504}-\frac{3347}{252} \nu-\frac{4127}{504} \nu^{2}\right) \\
& +S^{<i} v^{j>} \frac{\delta m}{m}\left(\frac{7}{9}+\frac{160}{63} \nu\right)(n v) \\
& +\Sigma^{<i} v^{j>}\left(\frac{131}{504}-\frac{4741}{504} \nu-\frac{5837}{504} \nu^{2}\right)(n v)
\end{aligned}
$$




$$
\begin{aligned}
& +(n S) v^{<i} v^{j>} \frac{\delta m}{m}\left(\frac{103}{126}-\frac{751}{252} \nu\right) \\
& \left.+(n \Sigma) v^{<i} v^{j>}\left(-\frac{275}{126}+\frac{1193}{252} \nu+\frac{691}{63} \nu^{2}\right)\right\} \\
& +\frac{G^{2} m^{2}}{r^{2}}\left\{(n S) n^{<i} n^{j>} \frac{\delta m}{m}\left(\frac{407}{126}-\frac{37}{126} \nu\right)+(n \Sigma) n^{<i} n^{j>}\left(\frac{155}{126}-\frac{311}{63} \nu-\frac{877}{252} \nu^{2}\right)\right. \\
& \left.\left.+S^{<i} n^{j>} \frac{\delta m}{m}\left(-\frac{103}{63}-\frac{25}{63} \nu\right)+\Sigma^{<i} n^{j>}\left(-\frac{275}{504}+\frac{3895}{504} \nu+\frac{1571}{504} \nu^{2}\right)\right\}\right] \\
& +\mathcal{O}\left(\frac{1}{c^{7}}\right) \\
& I_{S} i j k=\frac{r^{2} \nu}{c^{3}}\left\{\frac{9}{2} \frac{\delta m}{m}(\mathbf{S} \times \mathbf{v})^{<i} n^{j} n^{k>}+(\mathbf{\Sigma} \times \mathbf{v})^{<i} n^{j} n^{k>}\left(\frac{9}{2}-\frac{33}{2} \nu\right)\right. \\
& \left.+3 \frac{\delta m}{m}(\mathbf{n} \times \mathbf{S})^{<i} n^{j} v^{k>}+(\mathbf{n} \times \mathbf{\Sigma})^{<i} n^{j} v^{k>}(3-9 \nu)\right\} \\
& +\frac{r^{2} \nu}{c^{5}}\left[\left\{(\mathbf{S} \times \mathbf{v})^{<i} n^{j} n^{k>} \frac{\delta m}{m}\left(\frac{41}{20}-\frac{217}{20} \nu\right) v^{2}+(\boldsymbol{\Sigma} \times \mathbf{v})^{<i} n^{j} n^{k>}\left(\frac{41}{20}-21 \nu+\frac{203}{4} \nu^{2}\right) v^{2}\right.\right. \\
& +(\mathbf{n} \times \mathbf{S})^{<i} n^{j} v^{k>} \frac{\delta m}{m}\left(\frac{1}{5}-\frac{49}{10} \nu\right) v^{2}+(\mathbf{n} \times \mathbf{\Sigma})^{<i} n^{j} v^{k>}\left(\frac{1}{5}-\frac{11}{2} \nu+19 \nu^{2}\right) v^{2} \\
& +(\mathbf{S} \times \mathbf{v})^{<i} n^{j} v^{k>} \frac{\delta m}{m}\left(-\frac{1}{2}+\nu\right)(n v)+(\boldsymbol{\Sigma} \times \mathbf{v})^{<i} n^{j} v^{k>}\left(-\frac{1}{2}+\frac{9}{2} \nu-\frac{17}{2} \nu^{2}\right)(n v) \\
& +(n, S, v) n^{<i} v^{j} v^{k>} \frac{\delta m}{m}\left(\frac{7}{10}-\frac{7}{5} \nu\right)+(n, \Sigma, v) n^{<i} v^{j} v^{k>}\left(\frac{7}{10}-\frac{11}{2} \nu+\frac{19}{2} \nu^{2}\right) \\
& +(\mathbf{n} \times \mathbf{S})^{<i} v^{j} v^{k>} \frac{\delta m}{m}\left(\frac{4}{5}-\frac{8}{5} \nu\right)(n v)+(\mathbf{n} \times \boldsymbol{\Sigma})^{<i} v^{j} v^{k>}\left(\frac{4}{5}-4 \nu+4 \nu^{2}\right)(n v) \\
& \left.+(\mathbf{S} \times \mathbf{v})^{<i} v^{j} v^{k>} \frac{\delta m}{m}\left(\frac{7}{10}-\frac{7}{5} \nu\right)+(\mathbf{\Sigma} \times \mathbf{v})^{<i} v^{j} v^{k>}\left(\frac{7}{10}-\frac{9}{2} \nu+\frac{13}{2} \nu^{2}\right)\right\} \\
& +\frac{G m}{r}\left\{(n, S, v) n^{<i} n^{j} n^{k>} \frac{\delta m}{m}\left(\frac{139}{60}+\frac{131}{30} \nu\right)+(n, \Sigma, v) n^{<i} n^{j} n^{k>}\left(\frac{53}{20}-\frac{41}{4} \nu+\frac{9}{4} \nu^{2}\right)\right. \\
& +(\mathbf{n} \times \mathbf{S})^{<i} n^{j} n^{k>} \frac{\delta m}{m}\left(-\frac{17}{15}-\frac{479}{60} \nu\right)(n v)+(\mathbf{n} \times \mathbf{\Sigma})^{<i} n^{j} n^{k>}\left(-\frac{17}{15}-\frac{1}{12} \nu+\frac{52}{3} \nu^{2}\right)(n v) \\
& +(n S)(\mathbf{n} \times \mathbf{v})^{<i} n^{j} n^{k>} \frac{\delta m}{m}\left(\frac{9}{4}-\frac{3}{2} \nu\right)+(n \Sigma)(\mathbf{n} \times \mathbf{v})^{<i} n^{j} n^{k>}\left(\frac{9}{4}-\frac{33}{4} \nu+\frac{9}{4} \nu^{2}\right) \\
& +(\mathbf{S} \times \mathbf{v})^{<i} n^{j} n^{k>} \frac{\delta m}{m}\left(\frac{7}{6}+\frac{119}{12} \nu\right)+(\boldsymbol{\Sigma} \times \mathbf{v})^{<i} n^{j} n^{k>}\left(\frac{1}{6}+\frac{119}{12} \nu-\frac{253}{6} \nu^{2}\right) \\
& \left.\left.+(\mathbf{n} \times \mathbf{S})^{<i} n^{j} v^{k>} \frac{\delta m}{m}\left(\frac{269}{30}+\frac{257}{30} \nu\right)+(\mathbf{n} \times \mathbf{\Sigma})^{<i} n^{j} v^{k>}\left(\frac{89}{30}-\frac{19}{3} \nu-\frac{115}{6} \nu^{2}\right)\right\}\right] \\
& +\mathcal{O}\left(\frac{1}{c^{7}}\right)
\end{aligned}
$$




$$
\begin{aligned}
& J_{S} i j k=\frac{r^{2} \nu}{c}\left\{2 S^{<i} n^{j} n^{k>}+2 \frac{\delta m}{m} \Sigma^{<i} n^{j} n^{k>}\right\} \\
& +\frac{r^{2} \nu}{c^{3}}\left[\left\{S^{<i} n^{j} n^{k>}\left(\frac{5}{3}-5 \nu\right) v^{2}+\Sigma^{<i} n^{j} n^{k>} \frac{\delta m}{m}\left(\frac{5}{3}-\frac{25}{3} \nu\right) v^{2}\right.\right. \\
& +(S v) n^{<i} n^{j} v^{k>}\left(-\frac{5}{3}+5 \nu\right)+(\Sigma v) n^{<i} n^{j} v^{k>} \frac{\delta m}{m}\left(-\frac{5}{3}+\frac{19}{3} \nu\right) \\
& +S^{<i} n^{j} v^{k>}\left(-\frac{4}{3}+4 \nu\right)(n v)+\Sigma^{<i} n^{j} v^{k>} \frac{\delta m}{m}\left(-\frac{4}{3}+\frac{14}{3} \nu\right)(n v) \\
& +(n S) n^{<i} v^{j} v^{k>}\left(\frac{4}{3}-4 \nu\right)+(n \Sigma) n^{<i} v^{j} v^{k>} \frac{\delta m}{m}\left(\frac{4}{3}-\frac{14}{3} \nu\right) \\
& \left.+S^{<i} v^{j} v^{k>}\left(\frac{2}{3}-2 \nu\right)+\Sigma^{<i} v^{j} v^{k>} \frac{\delta m}{m}\left(\frac{2}{3}-\frac{4}{3} \nu\right)\right\} \\
& +\frac{G m}{r}\left\{(n S) n^{<i} n^{j} n^{k>}\left(\frac{16}{9}-\frac{16}{3} \nu\right)+(n \Sigma) n^{<i} n^{j} n^{k>} \frac{\delta m}{m}\left(\frac{4}{9}-\frac{11}{9} \nu\right)\right. \\
& \left.\left.+S^{<i} n^{j} n^{k>}\left(-\frac{10}{3}+6 \nu\right)+\Sigma^{<i} n^{j} n^{k>} \frac{\delta m}{m}\left(-\frac{10}{3}+\frac{11}{3} \nu\right)\right\}\right] \\
& +\mathcal{O}\left(\frac{1}{c^{5}}\right) \\
& I_{S} i j k l=\frac{r^{3} \nu}{c^{3}}\left\{(\mathbf{S} \times \mathbf{v})^{<i} n^{j} n^{k} n^{l>}\left(-\frac{32}{5}+\frac{96}{5} \nu\right)+(\boldsymbol{\Sigma} \times \mathbf{v})^{<i} n^{j} n^{k} n^{l>} \frac{\delta m}{m}\left(-\frac{32}{5}+\frac{84}{5} \nu\right)\right. \\
& \left.+(\mathbf{n} \times \mathbf{S})^{<i} n^{j} n^{k} v^{l>}\left(-\frac{24}{5}+\frac{72}{5} \nu\right)+(\mathbf{n} \times \boldsymbol{\Sigma})^{<i} n^{j} n^{k} v^{l>} \frac{\delta m}{m}\left(-\frac{24}{5}+\frac{48}{5} \nu\right)\right\} \\
& +\mathcal{O}\left(\frac{1}{c^{5}}\right) \\
& J_{S} i j k l=\frac{r^{3} \nu}{c}\left\{-\frac{5}{2} \frac{\delta m}{m} S^{<i} n^{j} n^{k} n^{l>}+\Sigma^{<i} n^{j} n^{k} n^{l>}\left(-\frac{5}{2}+\frac{15}{2} \nu\right)\right\}+\mathcal{O}\left(\frac{1}{c^{3}}\right) .
\end{aligned}
$$

We were able to perform a few technical tests on this calculation of the source multipole moments. We verified that the so-called "surface terms" can be computed either by a "bulk" integral over the entire three-dimensional space like other non-compact support terms, or by a surface integral extending on a sphere at spatial infinity. For such a test we have to use the alternative form of the blocks $\Sigma, \Sigma_{i}$ and $\Sigma_{i j}$ given in Eqs. (A1); we refer to Section IVD of Ref. [63] for a discussion of this type of terms. In addition we verified that certain quadratic non-compact support terms can be alternatively evaluated using some particular analytic kernels (denoted $Y_{L}, S_{L}$ and $T_{L}$ in Ref. [64]). Another, more physical, test of the expressions of the multipole moments we obtain before going to the CM frame, is the agreement with the so-called "boosted Kerr black hole limit" as investigated in the Appendix B. 


\section{Flux and orbital phasing for circular orbits}

For the spin-orbit effects at the post-Newtonian level considered in the present paper we can neglect all the corrections $\mathcal{O}\left(1 / c^{5}\right)$ in the relations between the canonical and source multipole moments, see Eqs. (2.4) and (2.5). Furthermore the relations between the radiative and canonical moments, Eqs. (2.3), imply a spin-orbit contribution due to gravitational wave tails and arising at the 3PN order; we ignore this contribution here since it has already been computed in Ref. [33], and since the next-to-leading tail contribution would enter the result at $4 \mathrm{PN}$ order only. Finally, for our present purpose, we can replace all the radiative moments $U_{L}$ and $V_{L}$ by the corresponding source moments $I_{L}$ and $J_{L}$ up to the 3.5PN spin-orbit level. We can therefore use for the flux (2.2) at that order the expression

$$
\begin{aligned}
\mathcal{F}=\frac{G}{c^{5}} & \left\{\frac{1}{5} I_{i j}^{(3)} I_{i j}^{(3)}+\frac{1}{c^{2}}\left[\frac{1}{189} I_{i j k}^{(4)} I_{i j k}^{(4)}+\frac{16}{45} J_{i j}^{(3)} J_{i j}^{(3)}\right]\right. \\
& \left.+\frac{1}{c^{4}}\left[\frac{1}{9072} I_{i j k l}^{(5)} I_{i j k l}^{(5)}+\frac{1}{84} J_{i j k}^{(4)} J_{i j k}^{(4)}\right]+\frac{1}{c^{6}}\left[\frac{4}{14175} J_{i j k l}^{(5)} J_{i j k l}^{(5)}\right]+(\text { tails })+\mathcal{O}\left(\frac{1}{c^{8}}\right)\right\} .
\end{aligned}
$$

The other terms do not contribute to the spin-orbit effect at the 3.5PN order. We insert the explicit results (3.10) for the source multipole moments into Eq. (3.11), we compute the time derivatives using systematically the equations of motion derived in Papers I \& II, and we specialize the result to the case of quasi-circular orbits, again using the material from Papers I \& II.

It is useful to introduce an orthonormal moving triad $\{\mathbf{n}, \boldsymbol{\lambda}, \boldsymbol{\ell}\}$ defined by $\mathbf{n}=\mathbf{x} / r$, $\boldsymbol{\ell}=\boldsymbol{L}_{\mathrm{N}} /\left|\boldsymbol{L}_{\mathrm{N}}\right|$ where $\boldsymbol{L}_{\mathrm{N}} \equiv m \nu \mathbf{x} \times \mathbf{v}$ denotes the Newtonian orbital angular momentum, and $\boldsymbol{\lambda}=\boldsymbol{\ell} \times \mathbf{n}$. Then the spin-orbit contributions in the flux will depend only on the projections of the spins perpendicular to the orbital plane, namely $S_{\ell} \equiv \boldsymbol{\ell} \cdot \mathbf{S}$ and $\Sigma_{\ell} \equiv \ell \cdot \Sigma$, where we recall that $\mathbf{S}$ and $\boldsymbol{\Sigma}$ are defined by Eqs. (3.9). Furthermore we denote the relevant post-Newtonian parameter for circular orbits by

$$
x=\left(\frac{G m \omega}{c^{3}}\right)^{2 / 3},
$$

where $\omega$ is the orbital frequency, related to the orbital separation $r$ by Eq. (4.2) in Paper II. We are then left with the main result of the present work, namely the spin-orbit contribution to the flux up to order $3.5 \mathrm{PN}$, as follows:

$$
\begin{aligned}
\mathcal{F}=\frac{32 c^{5}}{5 G} & x^{5} \nu^{2}\left(\frac{x^{3 / 2}}{G m^{2}}\right)\left\{-4 S_{\ell}-\frac{5}{4} \frac{\delta m}{m} \Sigma_{\ell}\right. \\
+ & x\left[\left(-\frac{9}{2}+\frac{272}{9} \nu\right) S_{\ell}+\left(-\frac{13}{16}+\frac{43}{4} \nu\right) \frac{\delta m}{m} \Sigma_{\ell}\right] \\
& +x^{3 / 2}\left[-16 \pi S_{\ell}-\frac{31 \pi}{6} \frac{\delta m}{m} \Sigma_{\ell}\right] \\
& +x^{2}\left[\left(\frac{476645}{6804}+\frac{6172}{189} \nu-\frac{2810}{27} \nu^{2}\right) S_{\ell}+\left(\frac{9535}{336}+\frac{1849}{126} \nu-\frac{1501}{36} \nu^{2}\right) \frac{\delta m}{m} \Sigma_{\ell}\right] \\
& \left.+\mathcal{O}\left(\frac{1}{c^{5}}\right)\right\} .
\end{aligned}
$$


We refer to Eq. (231) in [35] for the non-spin part of the energy flux up to the 3.5PN order. The tail-induced spin-orbit effect at 3PN order computed in Ref. [33] has also been added, but we recall that we neglect spin-spin interactions. We have checked that this result is in complete agreement in the test-mass limit where $\nu \rightarrow 0$ with the result of black-hole perturbation theory on a Kerr background obtained in Ref. 65.].

To obtain the evolution of the orbital phase for quasi-circular orbits we shall apply the energy conservation balance equation relating the flux $\mathcal{F}$ to the energy $E$ that is associated with the conservative part of the equations of motion:

$$
\frac{\mathrm{d} E}{\mathrm{~d} t}=-\mathcal{F} \text {. }
$$

Note that the balance equation (3.14) is valid in average over a long radiation-reaction time scale $\omega / \dot{\omega} \sim x^{-5 / 2}=\mathcal{O}\left(c^{5}\right)$; thus short periodic variations at the orbital frequency $\omega$ and at the spin precession frequencies $\omega_{\text {prec }} \sim x \omega$ have been averaged out. In order to apply the balance equation (3.14) we must ensure that the spins, or rather their projections $S_{1 \ell}$ and $S_{2 \ell}$ (or equivalently $S_{\ell}$ and $\Sigma_{\ell}$ ), are secularly constant over the radiation-reaction time scale $\omega / \dot{\omega}$.

This will be the case of the spin variables with conserved magnitude, as can be shown either explicitly at a given post-Newtonian order [66], or by the following structural argument valid at linear order in spins, extending the presentation of Ref. [33]. In the center-of-mass frame, the only vectors at our disposal, except for the spins, are $\mathbf{n}$ and $\mathbf{v}$. Recalling that the spin vectors are pseudovectors regarding parity transformation, we see that the only way spin-orbit contributions can enter scalars such as the energy $E$ or the flux $\mathcal{F}$ is through mixed products $\left(n, v, S_{1}\right)$ and $\left(n, v, S_{2}\right)$, i.e. through the components $S_{1 \ell}$ and $S_{2 \ell}$. Now, the same argument applies for the precession vectors $\Omega_{1,2}$ introduced in Eqs. (3.8): they must be pseudovectors, and, at linear order in spin, they must only depend on $\boldsymbol{n}$ and $\boldsymbol{v}$, so that we must have $\Omega_{1} \propto \ell$ and $\Omega_{2} \propto \ell$; this is explicitly seen for instance in Eq. (4.5) of Paper II. Now, the time derivative of the components along $\boldsymbol{\ell}$ of the spins are given by $\mathrm{d} S_{1 \ell} / \mathrm{d} t=\mathbf{S}_{1} \cdot\left[\mathrm{d} \boldsymbol{\ell} / \mathrm{d} t+\boldsymbol{\ell} \times \boldsymbol{\Omega}_{1}\right]$ and idem for 2 . The second term is zero, and since $\mathrm{d} \boldsymbol{\ell} / \mathrm{d} t=\mathcal{O}(S)$, we obtain that $S_{1 \ell}$ and $S_{2 \ell}$ are constants at linear order in the spins. This argument is valid at any post-Newtonian order and for general orbits, but is limited to spin-orbit terms.

The conservative energy $E$ has been obtained in Paper I and was reduced to circular orbits in Eq. (4.6) of Paper II. We recall here its expression:

$$
\begin{aligned}
\underset{S}{E}=- & \frac{m \nu c^{2} x}{2}\left(\frac{x^{3 / 2}}{G m^{2}}\right)\left\{\frac{14}{3} S_{\ell}+2 \frac{\delta m}{m} \Sigma_{\ell}\right. \\
+ & x\left[\left(11-\frac{61}{9} \nu\right) S_{\ell}+\left(3-\frac{10}{3} \nu\right) \frac{\delta m}{m} \Sigma_{\ell}\right] \\
+ & \left.x^{2}\left[\left(\frac{135}{4}-\frac{367}{4} \nu+\frac{29}{12} \nu^{2}\right) S_{\ell}+\left(\frac{27}{4}-39 \nu+\frac{5}{4} \nu^{2}\right) \frac{\delta m}{m} \Sigma_{\ell}\right]+\mathcal{O}\left(\frac{1}{c^{5}}\right)\right\} .
\end{aligned}
$$

See for instance Eq. (4.6) in Paper II for the complete expression of $E$ including non-spin terms. Applying now the balance equation (3.14), in which we can assume by the previous argument that the spin projections $S_{\ell}$ and $\Sigma_{\ell}$ are constant, one obtains the secular decrease of the orbital frequency as

$$
\left(\frac{\dot{\omega}}{\omega^{2}}\right)_{S}=\frac{96}{5} \nu x^{5 / 2}\left(\frac{x^{3 / 2}}{G m^{2}}\right)\left\{-\frac{47}{3} S_{\ell}-\frac{25}{4} \frac{\delta m}{m} \Sigma_{\ell}\right.
$$




$$
\begin{aligned}
+x[ & \left.\left(-\frac{5861}{144}+\frac{1001}{12} \nu\right) S_{\ell}+\left(-\frac{809}{84}+\frac{281}{8} \nu\right) \frac{\delta m}{m} \Sigma_{\ell}\right] \\
+x^{3 / 2} & {\left[-\frac{188 \pi}{3} S_{\ell}-\frac{151 \pi}{6} \frac{\delta m}{m} \Sigma_{\ell}\right] } \\
+x^{2} & {\left[\left(-\frac{4323559}{18144}+\frac{436705}{672} \nu-\frac{5575}{27} \nu^{2}\right) S_{\ell}\right.} \\
& \left.\left.+\left(-\frac{1195759}{18144}+\frac{257023}{1008} \nu-\frac{2903}{32} \nu^{2}\right) \frac{\delta m}{m} \Sigma_{\ell}\right]+\mathcal{O}\left(\frac{1}{c^{5}}\right)\right\} .
\end{aligned}
$$

Finally by a further integration we obtain the secular evolution of the orbital phase, or more precisely the so-called "carrier" phase defined by $\phi \equiv \int \omega \mathrm{d} t$, as

$$
\begin{aligned}
\phi_{S}=- & \frac{x^{-5 / 2}}{32 \nu}\left(\frac{x^{3 / 2}}{G m^{2}}\right)\left\{\frac{235}{6} S_{\ell}+\frac{125}{8} \frac{\delta m}{m} \Sigma_{\ell}\right. \\
+ & x \ln x\left[\left(-\frac{554345}{2016}-\frac{55}{8} \nu\right) S_{\ell}+\left(-\frac{41745}{448}+\frac{15}{8} \nu\right) \frac{\delta m}{m} \Sigma_{\ell}\right] \\
+x^{3 / 2} & {\left[\frac{940 \pi}{3} S_{\ell}+\frac{745 \pi}{6} \frac{\delta m}{m} \Sigma_{\ell}\right] } \\
+ & x^{2}\left[\left(-\frac{8980424995}{6096384}+\frac{6586595}{6048} \nu-\frac{305}{288} \nu^{2}\right) S_{\ell}\right. \\
& \left.\left.+\left(-\frac{170978035}{387072}+\frac{2876425}{5376} \nu+\frac{4735}{1152} \nu^{2}\right) \frac{\delta m}{m} \Sigma_{\ell}\right]+\mathcal{O}\left(\frac{1}{c^{5}}\right)\right\} .
\end{aligned}
$$

In the case of precessional binaries, for which the spins are not aligned or anti-aligned with the orbital angular momentum, the total phase $\Phi$ is the sum of the latter carrier phase and the precessional correction arising from the precession of the orbital plane, $\Phi=\phi+\phi_{\text {prec }}$. The precessional correction $\phi_{\text {prec }}$ can be computed numerically [13] or analytically (see for instance Ref. [33] for a computation at the $1 \mathrm{PN}$ order).

TABLE I. Spin-orbit contributions to the number of gravitational-wave cycles $\mathcal{N}_{\mathrm{GW}}=\left(\phi_{\max }-\right.$ $\left.\phi_{\min }\right) / \pi$ accumulated from $\omega_{\min }=\pi \times 10 \mathrm{~Hz}$ to $\omega_{\max }=\omega_{\mathrm{ISCO}}=c^{3} /\left(6^{3 / 2} \mathrm{Gm}\right)$ for binaries detectable by ground-based detectors LIGO and VIRGO. For each compact object we define the magnitude $\chi_{a}$ and the orientation $\kappa_{a}$ of the spin by $\mathbf{S}_{a} \equiv G m_{a}^{2} \chi_{a} \hat{\mathbf{S}}_{a}$ and $\kappa_{a} \equiv \hat{\mathbf{S}}_{a} \cdot \boldsymbol{\ell}$. For comparison, we give all the non-spin contributions up to $3.5 \mathrm{PN}$ order; however we neglect all the spin-spin terms. Notice that these figures are only indicative, and that the relative importance of the different terms changes only slightly when choosing another maximal frequency.

\begin{tabular}{|r|c|c|c|}
\hline & $1.4 M_{\odot}+1.4 M_{\odot}$ & $10 M_{\odot}+1.4 M_{\odot}$ & $10 M_{\odot}+10 M_{\odot}$ \\
\hline \hline Newtonian & 15952.6 & 3558.9 & 598.8 \\
$1 \mathrm{PN}$ & 439.5 & 212.4 & 59.1 \\
$1.5 \mathrm{PN}$ & $-210.3+65.6 \kappa_{1} \chi_{1}+65.6 \kappa_{2} \chi_{2}$ & $-180.9+114.0 \kappa_{1} \chi_{1}+11.7 \kappa_{2} \chi_{2}$ & $-51.2+16.0 \kappa_{1} \chi_{1}+16.0 \kappa_{2} \chi_{2}$ \\
$2 \mathrm{PN}$ & 9.9 & 9.8 & 4.0 \\
$2.5 \mathrm{PN}$ & $-11.7+9.3 \kappa_{1} \chi_{1}+9.3 \kappa_{2} \chi_{2}$ & $-20.0+33.8 \kappa_{1} \chi_{1}+2.9 \kappa_{2} \chi_{2}$ & $-7.1+5.7 \kappa_{1} \chi_{1}+5.7 \kappa_{2} \chi_{2}$ \\
$3 \mathrm{PN}$ & $2.6-3.2 \kappa_{1} \chi_{1}-3.2 \kappa_{2} \chi_{2}$ & $2.3-13.2 \kappa_{1} \chi_{1}-1.3 \kappa_{2} \chi_{2}$ & $2.2-2.6 \kappa_{1} \chi_{1}-2.6 \kappa_{2} \chi_{2}$ \\
$3.5 \mathrm{PN}$ & $-0.9+1.9 \kappa_{1} \chi_{1}+1.9 \kappa_{2} \chi_{2}$ & $-1.8+11.1 \kappa_{1} \chi_{1}+0.8 \kappa_{2} \chi_{2}$ & $-0.8+1.7 \kappa_{1} \chi_{1}+1.7 \kappa_{2} \chi_{2}$ \\
\hline
\end{tabular}


As a useful diagnosis to assess the importance of the latter spin effects, we have computed the number of accumulated gravitational-wave cycles between some minimal and maximal frequencies, corresponding to the bandwidth of ground-based detectors 7 The results are given in Table I. They show that the 3.5PN spin-orbit terms computed in the present paper can be numerically larger, for spins close to maximal and for suitable orientations, than the non-spin $3 \mathrm{PN}$ or $3.5 \mathrm{PN}$ contributions. We thus conclude that they are still relevant to be included in the gravitational wave templates of LIGO/VIRGO/LISA detectors for an accurate extraction of the binary parameters.

\section{ACKNOWLEDGEMENTS}

It is a pleasure to thank Guillaume Faye for discussions. A.B. is grateful for the support of the European Union FEDER funds, the Spanish Ministry of Economy and Competitiveness project FPA2010-16495 and the Conselleria d'Economia Hisenda i Innovacio of the Govern de les Illes Balears. Our computations were done using the package xAct, which handles symbolic tensor calculus within the software Mathematica ${ }^{\circledR}$ 67].

\section{Appendix A: Alternative expressions for $\Sigma, \Sigma_{i}, \Sigma_{i j}$}

In this Appendix we provide alternative expressions for the building blocks $\Sigma, \Sigma_{i}, \Sigma_{i j}$, defined by Eq. (2.7), which are useful for our practical computations. They are obtained by rewriting some products of derivatives so as to make Laplacians appear, for instance using $2 \partial_{i} A \partial_{i} B=\Delta(A B)-A \Delta B-B \Delta A$, and then using (2.14) to replace the Laplacians of elementary potentials by source terms. In this process, new compact-supported terms appear, proportional to $\sigma, \sigma_{i}, \sigma_{i j}$. We find

$$
\begin{aligned}
\Sigma=\sigma+\frac{4}{c^{4}} \sigma_{i i} V & +\frac{1}{c^{6}}\left(4 \hat{W}_{i j} \sigma_{i j}+8 \sigma_{i i} V^{2}+16 V_{i} V_{i} \sigma\right)-\frac{1}{2} \frac{1}{\pi G c^{2}} \Delta\left[V^{2}\right] \\
+\frac{1}{\pi G c^{4}}( & \left.-\frac{2}{3} \Delta\left[V^{3}\right]-\frac{1}{2} \Delta\left[V \hat{W}_{i i}\right]-\hat{W}_{i j} \partial_{j} \partial_{i} V+2 \partial_{i} V_{j} \partial_{j} V_{i}-\frac{1}{2}\left(\partial_{t} V\right)^{2}-2 V_{i} \partial_{t} \partial_{i} V\right) \\
+\frac{1}{\pi G c^{6}}( & -\frac{2}{3} \Delta\left[V^{4}\right]-\Delta\left[V^{2} \hat{W}_{i i}\right]+\frac{1}{2} \Delta\left[\hat{W}_{i j} \hat{W}_{i j}\right]-\frac{1}{4} \Delta\left[\hat{W}_{i i} \hat{W}_{j j}\right]-4 \Delta[V \hat{X}] \\
& -2 \Delta\left[V \hat{Z}_{i i}\right]-4 \hat{Z}_{i j} \partial_{j} \partial_{i} V+8 \partial_{i} V_{j} \partial_{j} \hat{R}_{i}-8 V_{i} \partial_{j} V_{i} \partial_{j} V+2 \partial_{t} V_{i} \partial_{t} V_{i} \\
& +4 \partial_{j} V_{i} \partial_{t} \hat{W}_{i j}-2 \partial_{i} V_{i} \partial_{t} \hat{W}_{j j}-4 \hat{R}_{i} \partial_{t} \partial_{i} V-2\left(\partial_{t} V\right)^{2} V-4 V_{i} \partial_{t} \partial_{i} V V \\
& \left.+\frac{1}{2} \partial_{t}^{2} \hat{W}_{i i} V-6 V_{i} \partial_{i} V \partial_{t} V-\partial_{t} \hat{W}_{i i} \partial_{t} V+\frac{1}{2} \hat{W}_{i i} \partial_{t}^{2} V\right)+\mathcal{O}\left(\frac{1}{c^{8}}\right), \\
\Sigma_{i}=\sigma_{i}+\frac{1}{c^{2}}( & \left.-2 V_{i} \sigma+2 V \sigma_{i}\right)+\frac{1}{c^{4}}\left(-4 \hat{R}_{i} \sigma+2 \hat{W}_{i j} \sigma_{j}+2 V^{2} \sigma_{i}+2 V_{i} \sigma_{j j}+2 V_{j} \sigma_{i j}\right) \\
+\frac{1}{\pi G c^{2}}( & \left.-\frac{1}{2} \Delta\left[V V_{i}\right]+\partial_{j} V \partial_{i} V_{j}+\frac{3}{4} \partial_{i} V \partial_{t} V\right)+\frac{1}{\pi G c^{4}}\left(-\Delta\left[\hat{R}_{i} V\right]-\frac{1}{2} \Delta\left[V^{2} V_{i}\right]\right. \\
& -\frac{1}{2} \Delta\left[V_{i} \hat{W}_{j j}\right]+\frac{1}{2} \Delta\left[V_{j} \hat{W}_{i j}\right]-V_{i} \partial_{j} V \partial_{j} V-\hat{W}_{j k} \partial_{k} \partial_{j} V_{i}+\partial_{j} \hat{W}_{i k} \partial_{k} V_{j}
\end{aligned}
$$

\footnotetext{
${ }^{7}$ Note however that the number of cycles of the carrier phase $\phi$ does not reflect the precession of the orbital plane, which has to be taken into account through $\Phi=\phi+\phi_{\text {prec }}$.
} 


$$
\begin{gathered}
+2 \partial_{j} V \partial_{i} \hat{R}_{j}+\frac{3}{2} V_{j} \partial_{j} V \partial_{i} V-\partial_{k} V_{j} \partial_{i} \hat{W}_{j k}+\frac{3}{2} V \partial_{i} V \partial_{t} V+\partial_{t} V \partial_{t} V_{i} \\
\left.+\partial_{j} V \partial_{t} \hat{W}_{i j}-2 V_{j} \partial_{t} \partial_{j} V_{i}+\frac{1}{2} V_{i} \partial_{t}^{2} V-\frac{1}{2} V \partial_{t}^{2} V_{i}\right)+\mathcal{O}\left(\frac{1}{c^{6}}\right), \\
\left.\Sigma_{i j}=\sigma_{i j}-\frac{1}{2} \delta^{i j} V \sigma+\frac{1}{c^{2}}\left(2 \delta^{i j} V_{k} \sigma_{k}-4 V_{(i} \sigma_{j}\right)+4 V \sigma_{i j}\right)+\frac{1}{\pi G}\left(-\frac{1}{16} \Delta\left[V^{2}\right] \delta^{i j}+\frac{1}{4} \partial_{i} V \partial_{j} V\right) \\
+\frac{1}{\pi G c^{2}}\left(-\frac{1}{2} \Delta\left[V_{i} V_{j}\right]+\frac{1}{4} \Delta\left[V_{k} V_{k}\right] \delta^{i j}-\frac{3}{8} \delta^{i j}\left(\partial_{t} V\right)^{2}-\frac{1}{2} \delta^{i j} \partial_{k} V_{l} \partial_{l} V_{k}+2 \partial_{k} V_{(i} \partial_{j)} V_{k}\right. \\
\left.-\partial_{i} V_{k} \partial_{j} V_{k}-\delta^{i j} \partial_{k} V \partial_{t} V_{k}+2 \partial_{(i} V \partial_{t} V_{j)}+\frac{1}{8} \delta^{i j} V \partial_{t}^{2} V\right)+\mathcal{O}\left(\frac{1}{c^{4}}\right) .
\end{gathered}
$$

Such a rewriting allows an independent (and also faster and easier) calculation for all the "Laplacian" terms appearing in the multipole moments, for which the integrals regularized by means of the Finite Part operation take a simple form in terms of an angular average at infinity (see the discussion in Section IVD of Ref. [63]).

\section{Appendix B: The boosted black hole limit}

In contrast to the case of the equations of motion for which we can perform several crucial verifications (see Paper I), there are not so many tests one can do in the case of the gravitational waveform and flux. In addition to the test-mass limit $\nu \rightarrow 0$ of the flux (3.13) which as we have seen perfectly recovers the result from Kerr black hole perturbations [65], we can perform another physical test directly at the level of the multipole moments, before the reduction to the center-of-mass frame: the so-called boosted black hole (BBH) limit [68].

The BBH is obtained in the limiting case when we suppress one of the two black holes (say 2) by setting its mass $m_{2}$ and spin $S_{2}^{i j}$ to be exactly zero into the general expressions of the multipole moments valid in an arbitrary frame, before going to the CM frame. What remains are then the multipole moments of a single Kerr black hole having mass $m_{1}$ and spin $S_{1}^{i j}$, and moving with constant velocity $v_{1}^{i}$. In the BBH limit the multipole moments should agree with those of a single Kerr black hole moving with constant velocity, i.e. a black hole space-time on which a special Lorentz transformation or boost has been applied. The BBH test is interesting because it verifies (although only partially) the global Lorentz invariance of the multipole moments and the radiation field [68].

We start with the Kerr metric in harmonic coordinates. Since we are interested in spinorbit effects we can work at linear order in the spin of the black hole. The "gothic" metric deviation $H^{\mu \nu}$ of the black hole in the rest frame associated with some harmonic coordinate system $X^{\mu}=(c T, \mathbf{X})$ (thus satisfying $\left.\partial_{\nu} H^{\mu \nu}=0\right)$ reads

$$
\begin{aligned}
H^{00} & =1-\frac{\left(1+\frac{G M}{c^{2} R}\right)^{3}}{1-\frac{G M}{c^{2} R}}, \\
H^{0 i} & =-\frac{2 G}{c^{4} R^{2}} \frac{\varepsilon^{i j k} \tilde{\mathcal{S}}_{j} N_{k}}{1-\frac{G M}{c^{2} R}}+\mathcal{O}\left(\tilde{\mathcal{S}}^{2}\right), \\
H^{i j} & =-\frac{G^{2} M^{2}}{c^{4} R^{2}} N^{i} N^{j}+\frac{2 G^{2} M}{c^{6} R^{3}} N^{(i} \varepsilon^{j) k l} \tilde{\mathcal{S}}_{k} N_{l}+\mathcal{O}\left(\tilde{\mathcal{S}}^{2}\right),
\end{aligned}
$$


where $M$ is the mass of the black hole and $\tilde{\mathcal{S}}_{i}$ is the spin vector of the black hole in the rest frame. For convenience in this Appendix we define the spin vector directly from the spin tensor as $\tilde{S}_{i} \equiv \frac{1}{2} \varepsilon_{i j k} S^{j k}$. In the rest frame of the black hole we denote the spin tensor by $\mathcal{S}^{i j}$ and the spin vector appearing in Eqs. (B1) is defined by $\tilde{\mathcal{S}}_{i} \equiv \frac{1}{2} \varepsilon_{i j k} \mathcal{S}^{j k}$. We also denote the radial distance and unit direction in the rest frame by $R \equiv|\mathbf{X}|$ and $N^{i} \equiv X^{i} / R$.

Following Ref. [68] we apply a global boost $\Lambda^{\mu}{ }_{\nu}(\mathbf{V})$ with constant velocity $\mathbf{V}=\left(V^{i}\right)$. In our conventions we pose

$$
\begin{aligned}
\Lambda_{0}^{0}(\mathbf{V}) & =\gamma, \\
\Lambda_{0}^{i}(\mathbf{V}) & =\Lambda^{0}{ }_{i}(\mathbf{V})=\gamma \frac{V^{i}}{c}, \\
\Lambda_{j}^{i}(\mathbf{V}) & =\delta_{j}^{i}+\frac{\gamma^{2}}{\gamma+1} \frac{V^{i} V_{j}}{c^{2}},
\end{aligned}
$$

with $\gamma \equiv\left(1-\frac{V^{2}}{c^{2}}\right)^{-1 / 2}$. In the global frame defined by $x^{\mu}=\Lambda^{\mu}{ }_{\nu} X^{\nu}$ the Kerr black hole metric will then be given by

$$
h^{\mu \nu}(x)=\Lambda_{\rho}^{\mu} \Lambda_{\sigma}^{\nu} H^{\rho \sigma}\left(\Lambda^{-1} x\right),
$$

where $\left(\Lambda^{-1}\right)^{\mu}{ }_{\nu}=\Lambda_{\nu}{ }^{\mu}$ denotes the inverse Lorentz transformation. The radial distance $r$ and unit direction $n^{i}$ in the global frame $x^{\mu}=(c t, \mathbf{x})$ are related to the rest-frame counterparts $R$ and $N^{i}$ by

$$
\begin{aligned}
R & =r\left[1+c^{2}\left(\gamma^{2}-1\right)\left(\frac{t}{r}\right)^{2}-2 \gamma^{2}(V n)\left(\frac{t}{r}\right)+\gamma^{2} \frac{(V n)^{2}}{c^{2}}\right]^{1 / 2}, \\
N^{i} & =\frac{r}{R}\left[n^{i}-\gamma V^{i}\left(\frac{t}{r}\right)+\frac{\gamma^{2}}{\gamma+1} \frac{V^{i}}{c^{2}}(V n)\right] .
\end{aligned}
$$

See [68]; we denote the usual Euclidean scalar product by $(V n) \equiv V^{i} n^{i}$. In addition the spin must also be transformed and we find that the spin vector $\tilde{\mathcal{S}}_{i}$ in the rest frame is related to the spin vector $\tilde{S}_{i}$ in the global frame by

$$
\tilde{\mathcal{S}}_{i}=\tilde{S}_{i}-\frac{\gamma}{\gamma+1} \frac{(\tilde{S} V)}{c^{2}} V_{i}
$$

Notice that the spin vector $\tilde{S}_{i}$ we are using here should rather be viewed as a covector, since it agrees with the spatial components of the covariant vector $\tilde{S}_{\mu}$ satisfying $u^{\mu} \tilde{S}_{\mu}=0$, i.e. such that $\tilde{\mathcal{S}}_{0}=0$ in the rest frame of the black hole. If one were to use instead the spin vector $S_{i}$ with conserved magnitude (as we did in all of this paper), one should have to apply a correction which is given at the $2 \mathrm{PN}$ order and for the $\mathrm{BBH}$ case by

$$
\tilde{S}_{i}=S_{i}+\frac{(S V)}{c^{2}}\left(\frac{1}{2}+\frac{3}{8} \frac{V^{2}}{c^{2}}\right) V_{i}
$$

We now compute all the required multipole moments of the BBH by inserting the boosted Kerr metric (B1) $-(\bar{B} 3)$ into the general definitions of the source multipole moments. However this calculation is not straightforward starting from the defining expressions (2.6) of the source multipole moments. Instead it was found in Ref. [68] that the best is to use some 
different expressions of the multipole moments, entirely given by surface integrals at spatial infinity; they have been derived in Eqs. (2.19) of [68], see also (2.29) for the practical implementation. In the present paper we have used the same expressions of the multipole moments and inserted there the BBH solution (B1)-(B3). Expanding the results at the required post-Newtonian order, and keeping only the spin parts of the multipole moments, we obtain:

$$
\begin{aligned}
I_{\mathrm{S}} & =\frac{t}{c^{3}}(\tilde{\mathbf{S}} \times \mathbf{V})_{<i} V_{j>}\left[-\frac{4}{3}-\frac{6}{7} \frac{V^{2}}{c^{2}}-\frac{83}{126} \frac{V^{4}}{c^{4}}\right]+\mathcal{O}\left(\frac{1}{c^{9}}\right), \\
J_{\mathrm{S}} & =\frac{t}{c} \tilde{S}_{<i} V_{j>}\left[\frac{3}{2}+\frac{17}{28} \frac{V^{2}}{c^{2}}+\frac{149}{336} \frac{V^{4}}{c^{4}}\right] \\
& +\frac{t}{c^{3}} V_{<i} V_{j>}(\tilde{S} V)\left[-\frac{8}{7}-\frac{2}{3} \frac{V^{2}}{c^{2}}\right]+\mathcal{O}\left(\frac{1}{c^{7}}\right), \\
I_{\mathrm{S}} & =\frac{t^{2}}{c^{3}}(\tilde{\mathbf{S}} \times \mathbf{V})_{<i} V_{j} V_{k>}\left[-\frac{3}{2}-\frac{5}{4} \frac{V^{2}}{c^{2}}\right]+\mathcal{O}\left(\frac{1}{c^{7}}\right), \\
J_{\mathrm{S}}{ }_{i j k} & =\frac{t^{2}}{c} \tilde{S}_{<i} V_{j} V_{k>}\left[2+\frac{V^{2}}{c^{2}}\right]-\frac{4}{3} \frac{t^{2}}{c^{3}} V_{<i} V_{j} V_{k>}(\tilde{S} V)+\mathcal{O}\left(\frac{1}{c^{5}}\right), \\
I_{\mathrm{S}} i j k l & =-\frac{8}{5} \frac{t^{3}}{c^{3}}(\tilde{\mathbf{S}} \times \mathbf{V})_{<i} V_{j} V_{k} V_{l>}+\mathcal{O}\left(\frac{1}{c^{5}}\right), \\
\left.J_{\mathrm{S}}\right) & \frac{5}{2} \frac{t^{3}}{c} \tilde{S}_{<i} V_{j} V_{k} V_{l>}+\mathcal{O}\left(\frac{1}{c^{3}}\right) .
\end{aligned}
$$

These results are in perfect agreement with those obtained directly from our general computation of the multipole moments of black hole binaries, by setting $m_{2}=0$ and $\tilde{S}_{2 i}=0$, and making the identifications $m_{1} \equiv M, \tilde{S}_{1 i} \equiv \tilde{S}_{i}, y_{1}^{i} \equiv V^{i} t$ and $v_{1}^{i} \equiv V^{i}$.

[1] S. Marsat, A. Bohé, G. Faye, and L. Blanchet, Class. Quant. Grav. (2012), gr-qc/1210.4143.

[2] A. Bohé, S. Marsat, G. Faye, and L. Blanchet, Class. Quant. Grav. (2012), gr-qc/1212.5520.

[3] M. A. Abramowicz and W. Kluźniak, Astron. Astrophys. 374, L19 (2001).

[4] T. E. Strohmayer, Astrophys. J. 552, L49 (2001).

[5] J. E. McClintock, R. Shafee, R. Narayan, R. A. Remillard, S. W. Davis, and L.-X. Li, Astrophys. J. 652, 518 (2006), arXiv:astro-ph/0606076.

[6] L. Gou, J. E. McClintock, M. J. Reid, J. A. Orosz, J. F. Steiner, R. Narayan, J. Xiang, R. A. Remillard, K. A. Arnaud, and S. W. Davis, Astrophys. J. 742, 85 (2011), arXiv:1106.3690 [astro-ph.HE].

[7] M. A. Nowak, J. Wilms, K. Pottschmidt, N. Schulz, D. Maitra, and J. Miller, Astrophys. J. 744, 107 (2012), arXiv:1109.6008.

[8] A. C. Fabian and G. Miniutti, in The Kerr Spacetime: Rotating Black Holes in General Relativity, edited by D. L. Wiltshire, M. Visser, and S. M. Scott (Cambridge U. Press, 2009) Chap. 9, arXiv:astro-ph/0507409.

[9] L. W. Brenneman and C. S. Reynolds, Astrophys. J. 652, 1028 (2006), arXiv:astro-ph/0608502,

[10] L. W. Brenneman, C. S. Reynolds, M. A. Nowak, R. C. Reis, M. Trippe, A. C. Fabian, K. Iwa- 
sawa, J. C. Lee, J. M. Miller, R. F. Mushotzky, K. Nandra, and M. Volonteri, Astrophys. J. 736, 103 (2011), arXiv:1104.1172 [astro-ph.HE].

[11] C. Cutler, T. Apostolatos, L. Bildsten, L. Finn, E. Flanagan, D. Kennefick, D. Markovic, A. Ori, E. Poisson, G. Sussman, and K. Thorne, Phys. Rev. Lett. 70, 2984 (1993).

[12] C. Cutler and E. Flanagan, Phys. Rev. D 49, 2658 (1994).

[13] T. Apostolatos, C. Cutler, G. Sussman, and K. Thorne, Phys. Rev. D 49, 6274 (1994).

[14] B. Barker and R. O'Connell, Phys. Rev. D 12, 329 (1975).

[15] B. Barker and R. O'Connell, Gen. Relativ. Gravit. 11, 149 (1979).

[16] L. Kidder, C. Will, and A. Wiseman, Phys. Rev. D 47, R4183 (1993).

[17] L. Kidder, Phys. Rev. D 52, 821 (1995), gr-qc/9506022.

[18] W. D. Goldberger and I. Z. Rothstein, Phys. Rev. D 73, 104029 (2006), gr-qc/0409156.

[19] R. Porto, Phys. Rev. D 73, 104031 (2006), gr-qc/0511061.

[20] H. Tagoshi, A. Ohashi, and B. Owen, Phys. Rev. D 63, 044006 (2001).

[21] L. Blanchet, A. Buonanno, and G. Faye, Phys. Rev. D 74, 104034 (2006), erratum Phys. Rev. $D, 75: 049903,2007$, gr-qc/0605140.

[22] T. Damour, P. Jaranowski, and G. Schäfer, Phys. Rev. D 77, 064032 (2008).

[23] M. Levi, Phys.Rev. D82, 104004 (2010), arXiv:1006.4139 [gr-qc].

[24] R. A. Porto, Classical and Quantum Gravity 27, 205001 (2010), arXiv:1005.5730 [gr-qc],

[25] S. Hergt, J. Steinhoff, and G. Schäfer, Class. Quant. Grav. 27, 135007 (2010), arXiv:1002.2093 [gr-qc],

[26] J. Steinhoff, S. Hergt, and G. Schäfer, Phys. Rev. D 78, 101503 (2008).

[27] J. Hartung and J. Steinhoff, Annalen der Physik 523, 919 (2011), arXiv:1107.4294 [gr-qc]

[28] R. A. Porto and I. Z. Rothstein, Phys.Rev. D78, 044012 (2008), arXiv:0802.0720 [gr-qc],

[29] R. A. Porto and I. Z. Rothstein, Phys.Rev. 78, 044013 (2008), arXiv:0804.0260 [gr-qc],

[30] J. Hartung and J. Steinhoff, Annalen der Physik 523, 783 (2011), arXiv:1104.3079 [gr-qc],

[31] J. Hartung, J. Steinhoff, and G. Schäfer, Annalen der Physik (2013), arXiv:1302.6723 [gr-qc].

[32] B. Owen, H. Tagoshi, and A. Ohashi, Phys. Rev. D 57, 6168 (1998).

[33] L. Blanchet, A. Buonanno, and G. Faye, Phys.Rev. D84, 064041 (2011) arXiv:1104.5659 [gr-qc],

[34] R. A. Porto, A. Ross, and I. Z. Rothstein, JCAP 1103, 009 (2011), arXiv:1007.1312 [gr-qc],

[35] L. Blanchet, Living Rev. Rel. 9, 4 (2006), gr-qc/0202016.

[36] L. Blanchet and T. Damour, Phil. Trans. Roy. Soc. Lond. A 320, 379 (1986).

[37] L. Blanchet, Proc. Roy. Soc. Lond. A 409, 383 (1987).

[38] L. Blanchet and T. Damour, Phys. Rev. D 46, 4304 (1992).

[39] L. Blanchet, Phys. Rev. D 47, 4392 (1993).

[40] L. Blanchet and T. Damour, Annales Inst. H. Poincaré Phys. Théor. 50, 377 (1989).

[41] L. Blanchet, Phys. Rev. D 51, 2559 (1995), gr-qc/9501030.

[42] L. Blanchet, Class. Quant. Grav. 15, 113 (1998), erratum Class. Quant. Grav., 22:3381, 2005, gr-qc/9710038.

[43] L. Blanchet, Class. Quant. Grav. 15, 1971 (1998), gr-qc/9801101.

[44] O. Poujade and L. Blanchet, Phys. Rev. D 65, 124020 (2002), gr-qc/0112057.

[45] M. Mathisson, General Relativity and Gravitation 42, 1011 (2010).

[46] A. Papapetrou, Proc. Phys. Soc. A 64, 57 (1951).

[47] A. Papapetrou, Proc. R. Soc. London A 209, 248 (1951).

[48] E. Corinaldesi and A. Papapetrou, Proc. R. Soc. London A 209, 259 (1951).

[49] W. Tulczyjew, Bull. Acad. Polon. Sci. 5, 279 (1957). 
[50] W. Tulczyjew, Acta Phys. Polon. 18, 37 (1959).

[51] A. Trautman, Gen. Relat. Grav. 34, 721 (2002), reprinted from lectures delivered in 1958.

[52] W. G. Dixon, Il Nuovo Cimento 34, 317 (1964).

[53] W. G. Dixon, General Relativity and Gravitation 4, 199 (1973).

[54] W. G. Dixon, in Isolated Gravitating Systems in General Relativity: Proceedings of the International School of Physics "Enrico Fermi"; course 67, edited by J. Ehlers (1979) pp. 156-219.

[55] I. Bailey and W. Israel, Ann. Phys. 130, 188 (1980).

[56] L. Blanchet and G. Faye, J. Math. Phys. 41, 7675 (2000), gr-qc/0004008.

[57] L. Blanchet, T. Damour, and G. Esposito-Farèse, Phys. Rev. D 69, 124007 (2004), gr-qc/0311052.

[58] J. Hadamard, Le problème de Cauchy et les équations aux dérivées partielles linéaires hyperboliques (Hermann, Paris, 1932).

[59] I. M. Gel'fand and G. E. Shilov, Generalized functions (Academic Press, New York, 1964).

[60] L. Schwartz, Théorie des distributions (Hermann, Paris, 1978).

[61] K. Thorne, Rev. Mod. Phys. 52, 299 (1980).

[62] L. Blanchet, G. Faye, B. R. Iyer, and S. Sinha, Class. Quant. Grav. 25, 165003 (2008).

[63] L. Blanchet and B. R. Iyer, Phys. Rev. D 71, 024004 (2004), gr-qc/0409094.

[64] L. Blanchet, B. R. Iyer, and B. Joguet, Phys. Rev. D 65, 064005 (2002), erratum Phys. Rev. D, 71:129903(E), 2005, gr-qc/0105098.

[65] H. Tagoshi, M. Shibata, T. Tanaka, and M. Sasaki, Phys. Rev. D 54, 1439 (1996).

[66] C. Will, Phys. Rev. D 71, 084027 (2005), gr-qc/0502039.

[67] J. M. Martín-García, A. García-Parrado, A. Stecchina, B. Wardell, C. Pitrou, D. Brizuela, D. Yllanes, G. Faye, L. Stein, R. Portugal, and T. Bäckdahl, "xAct: Efficient tensor computer algebra for Mathematica," (GPL 2002-2013), http://www.xact.es/.

[68] L. Blanchet, T. Damour, and B. R. Iyer, Class. Quant. Grav. 22, 155 (2005), gr-qc/0410021. 